\title{
Qualitative and quantitative evaluation of thylakoid complexes separated by Blue Native PAGE
}

\author{
Éva Sárvári1 ${ }^{*}$, Gabriella Gellén ${ }^{2}$, Máté Sági-Kazár ${ }^{1,3}$, Gitta Schlosser ${ }^{2}$, Katalin Solymosi ${ }^{4}$ and Ádám Solti ${ }^{1}$ (i)
}

\begin{abstract}
Background: Blue Native polyacrylamide gel electrophoresis (BN PAGE) followed by denaturing PAGE is a widely used, convenient and time efficient method to separate thylakoid complexes and study their composition, abundance, and interactions. Previous analyses unravelled multiple monomeric and dimeric/oligomeric thylakoid complexes but, in certain cases, the separation of complexes was not proper. Particularly, the resolution of super- and megacomplexes, which provides important information on functional interactions, still remained challenging.

Results: Using a detergent mixture of $1 \%(\mathrm{~W} / \mathrm{N}) n$-dodecyl- $\beta$-D-maltoside plus $1 \%(\mathrm{~W} / \mathrm{V})$ digitonin for solubilisation and $4.3-8 \%$ gel gradients for separation as methodological improvements in BN PAGE, several large photosystem (PS) I containing bands were detected. According to BN(/BN)/SDS PAGE and mass spectrometry analyses, these PSI bands proved to be PSI-NADH dehydrogenase-like megacomplexes more discernible in maize bundle sheath thylakoids, and PSI complexes with different light-harvesting complex (LHC) complements (PSI-LHCII, PSI-LHCII*) more abundant in mesophyll thylakoids of lincomycin treated maize. For quantitative determination of the complexes and their comparison across taxa and physiological conditions, sample volumes applicable to the gel, correct baseline determination of the densitograms, evaluation methods to resolve complexes running together, calculation of their absolute/relative amounts and distribution among their different forms are proposed.
\end{abstract}

Conclusions: Here we report our experience in Blue/Clear-Native polyacrylamide gel electrophoretic separation of thylakoid complexes, their identification, quantitative determination and comparison in different samples. The applied conditions represent a powerful methodology for the analysis of thylakoid mega- and supercomplexes.

Keywords: Bundle sheath, Blue/Clear Native PAGE, Chloroplast NADH dehydrogenase-like complex, Lincomycin, Maize, Mesophyll, Photosystem I, Populus

\section{Background}

In oxygenic photosynthesis, the conversion of the energy of sunlight into chemical energy is carried out by the multi-subunit complexes of photosynthetic membranes. All these complexes, the photosystem (PS) I and

\footnotetext{
*Correspondence: eva.sarvari@ttk.elte.hu

${ }^{1}$ Department of Plant Physiology and Molecular Plant Biology, Institute of Biology, Faculty of Science, ELTE Eötvös Loránd University, Pázmány Péter sétány 1/C, Budapest 1117, Hungary

Full list of author information is available at the end of the article
}

PSII with their light-harvesting complexes (Lhc/LHC), the cytochrome (Cyt) $b_{6} f$ complex, chloroplast NADH dehydrogenase-like complex (NDH), and ATP synthase (ATPs), are composed of numerous polypeptides many of which are highly hydrophobic $[1,2]$. To understand the regulation of photosynthesis, it is important to follow qualitative and quantitative changes in thylakoid composition under different circumstances. To this purpose, these components can be examined by different denaturing and native polyacrylamide gel electrophoresis (PAGE) methods. Two-dimensional PAGE using isoelectric 
focusing in the first dimension and sodium dodecyl sulphate (SDS) PAGE in the second dimension, separating the proteins by both charge and size, gives high resolution. However, it is often difficult to rehydrate and solubilise delipidated hydrophobic membrane proteins, thus it cannot be easily used for the investigation of changes in the quantitative aspects of thylakoid proteome [3]. Native separation methods are, indeed, more convenient. Green PAGE techniques [4-8] apply non-denaturing non-ionic detergents for solubilisation but proper resolution cannot be achieved without the presence of a small amount of ionic detergent that gives charge to hydrophobic proteins. Nevertheless, mega- and supercomplexes of thylakoids as well as association of LHCII antennae are very sensitive to the presence of most ionic detergents that generally leads to the loss of multiple complex interactions. In addition, even sophisticated and mild gradient centrifugation $[9,10]$ does not allow the separation of intact complexes with high resolution in one step. At the same time, Blue Native (BN) PAGE introduced by Schägger and von Jagow [11] applied in both one- and two-dimensional separations gives superior quality of resolution of membrane complexes, the supramolecular organisation of which is well retained [3, 12-17]. In the application of BN PAGE, after gentle solubilisation of membranes, anionic dye Coomassie Brilliant Blue G-250 is added to the samples. It binds, on a non-covalent way, to the surface of both hydrophilic and hydrophobic residues providing a negative charge that enhances migration of complexes towards the anode and hampers aggregation but does not influence the protein-protein interactions. Thus, separation of complexes by BN PAGE allows obtaining structural information about the native state of the thylakoid complexome in a time efficient way in one separation step.

BN PAGE has been widely used to compare thylakoid complexes in different plant species $[18,19]$, to follow their changes during development [20,21], acclimation to various environments [22-25], under stress circumstances [26-31], and in mutant plants [32-35]. In some cases, a useful variant of BN PAGE, Clear Native $(\mathrm{CN})$ PAGE, showing similar band pattern to BN PAGE, was also applied [16, 36]. Mega- and supercomplexes are better resolved by large-pore BN-PAGE $[16,17,22]$. Nowadays, complexome profiling, i.e. mass spectrometry (MS) determination of polypeptide content of bands cut out of lanes or obtained by slicing the lanes, offers great possibilities for discovering new bands and new proteins in the band pattern but requires large-scale MS capacity [37].

Previous analyses unravelled multiple monomeric and dimeric/oligomeric thylakoid complexes. The monomers and PSII supercomplexes (PSII-s) are well characterised [9, 17, 38]. PSII-s differing in antenna size are $\mathrm{C}_{2} \mathrm{~S}_{2} \mathrm{M}_{2}, \mathrm{C}_{2} \mathrm{~S}_{2} \mathrm{M}, \mathrm{C}_{2} \mathrm{~S}_{2}, \mathrm{C}_{2} \mathrm{~S}$, where $\mathrm{C}, \mathrm{S}$ and $\mathrm{M}$ are standing for PSII core, strongly (via Lhcb5) and moderately bound (via Lhcb4 and Lhcb6) LHCII trimers building of Lhcb1,2 and Lhcb1,3, respectively. The monomer PSI complex is built of PSI core components and LHCI composed of Lhca pigment-proteins organised in dimers (Lhca1,4 and Lhca2,3), and arranged in a semicircle around the core between PsaG and PsaK [39]. The PSI may also bind additional LHCII trimer on the opposite side of the complex (known as PSI-LHCII complex), and this LHCII is highly efficient in light harvesting for PSI and thus important in excitation energy allocation during light acclimation process [10,39-41]. More LHCII trimers connected to PSI were discovered using milder methods for solubilisation [42-45]. The composition of PSI bands larger than PSI-LHCII obtained by BN PAGE, however, is not totally clear, though several megacomplex $(\mathrm{mc})$ and supercomplex bands consisting of only PSI or also containing PSII and/or LHCII were reported in Arabidopsis and other higher plant thylakoids [16, 17, 22, 36, 38, 46]. Although PSI-Cyt $b_{6} f$ or PSI-NDH complexes involved either in cyclic electron flow or chlororespiration, respectively, were isolated [47] or detected by single particle analysis [43, 48], only PSI-NDH complex could be separated by BN PAGE [16, 37, 49, 50].

The primary aim of this paper is to show the qualitative and quantitative evaluation of $\mathrm{BN}-/ \mathrm{CN}$ PAGE patterns of poplar and maize thylakoid models. As a powerful technique, here we report the way of retaining the native state of mega-/supercomplexes by solubilisation with $1 \%(\mathrm{w} / \mathrm{V}) n$-dodecyl- $\beta$-D-maltoside $(\beta$-DM) plus $1 \%(\mathrm{w} / \mathrm{V})$ digitonin mixture and increasing the resolution of the large complex region of BN PAGE using $4.3-8 \%$ gel gradient, which is employed for the detection of several PSI mega/supercomplexes in untreated bundle sheath (BS), and lincomycin (LM) treated mesophyll (M) thylakoids. We demonstrate the quantitative comparison of complexes in untreated and LM treated $M$ thylakoids involving evaluation methods to show how complexes running together can be resolved, and how their absolute/relative amounts and distribution among their different forms can be calculated.

\section{Results and discussion}

To follow the variations of thylakoid composition in different samples and under various conditions, it is necessary to extract the complexes in their native form, separate them as clean bands identified by their polypeptide composition and to assess changes in their quantities. 


\section{Evaluation of gel patterns}

As a frequently used system, we applied short gels for BN PAGE [Mini-Protean II Bio-Rad apparatus] as it was advised to save reagents and achieve a faster and milder separation [16]. Long gels are not so beneficial because the bands sometimes are less sharp and the resolution of bands is usually not better than in short gels [27, 51]. However, slightly longer gels than the ones used in Mini-Protean II may be more convenient. In the second or third dimensional SDS PAGE separations of polypeptides we used Laemmli gels [52] with glycerol which gives sharp bands/spots (Additional file 1: Fig. S1b, d) and quite good resolution both in the high and low molecular mass region. However, Tricine-SDS PAGE [53] or SDS gels containing urea [16] are used the most frequently because of their higher resolution in the low molecular mass region, and better separation of Lhc apoproteins. Robust methods should operate across taxon borders. Since starting trials of our BN PAGE investigations were performed on poplar thylakoids, evaluation of gel pattern will be demonstrated on this model.

\section{Identification of thylakoid complexes}

Identification of thylakoid complexes are based on their characteristic polypeptide composition (Fig. 1a) determined as in Basa et al. [28]. Otherwise, in angiosperms, complexes separated by BN PAGE were qualitatively similar apart from variations in abundance in the different species (Additional file 1: Fig. S2). PSI core monomers together with LHCI (Lhca1-4) were found in PSI and depending on the detergents used also in PSI-LHCII (binding a trimeric LHCII) bands. PSII dimer (PSII-d: $\mathrm{C}_{2}$ ) showed very similar mobility to PSI. Interestingly, another PSII complex running a little further than PSII$\mathrm{d}$ and containing some LHCII, probably CS [9], was also detectable in poplar. Components of PSII were also present in PSII supercomplexes, PSII monomer (PSII-m: C), and chlorophyll-protein-43 (CP43)-less PSII (only a very faint band in poplar). Free Lhc bands occurred as LHCII assembly (LHCII-a) containing LHCII trimer (LHCIIt) together with CP29 and CP24, LHCII-t, and Lhc-m. LHCI was strongly bound to PSI core, so Lhca was rarely found in the Lhc-m band. The PSI subcomplexes and LHCII-t bands contained some residual bound chlorophyll (Chl) even after the denaturing separation (see for maize in Additional file 1: Fig. S1). Except for a few cases, the Cyt $b_{6} f$-d did not separate well from PSII-m. In the case of stronger solubilisation, a Cyt $b_{\sigma} f$-m band also appeared below the LHCII-t band. Regarding the ATPs complex, most coupling factor $1\left(\mathrm{CF}_{1}\right)$ was washed out from the surface of thylakoids [52] to be able to solubilise the thylakoids by lower amounts of detergents.

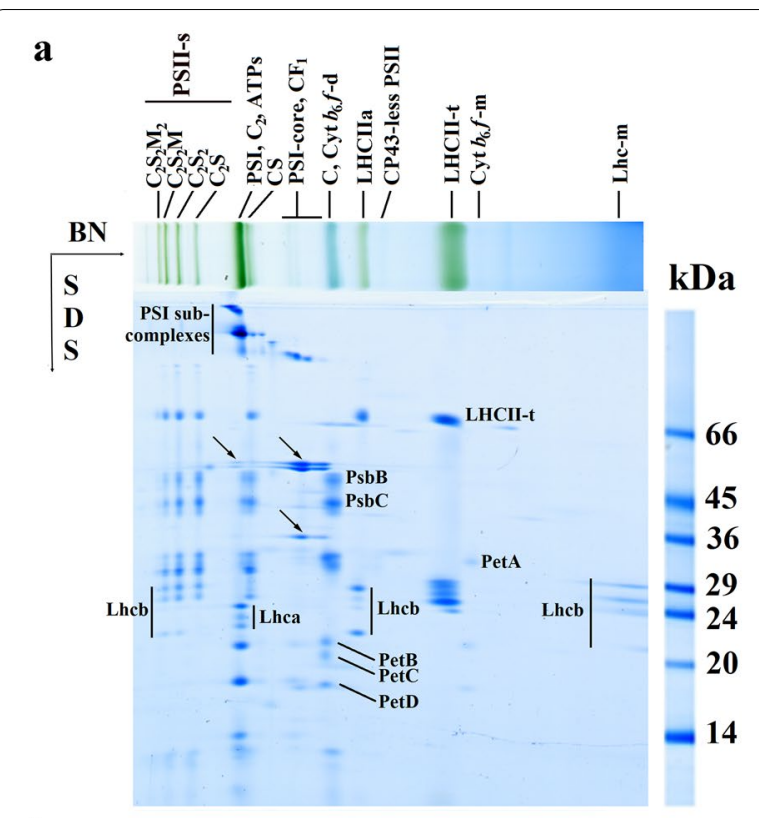

b
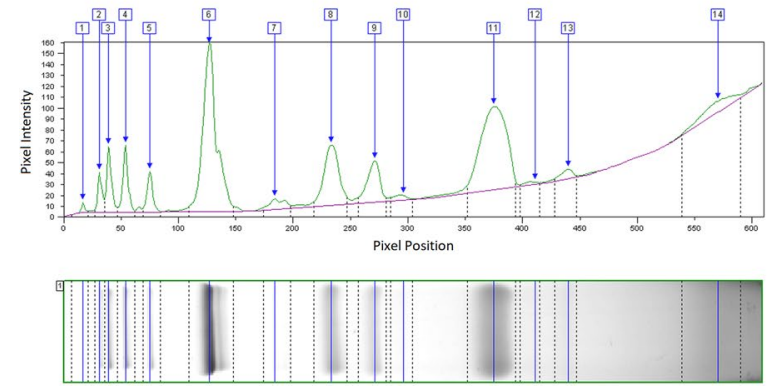

Fig. 1 BN/SDS PAGE pattern of poplar (Populus jacquemontiana var. glauca) thylakoid complexes. a Thylakoids $\left(500 \mu \mathrm{g} \mathrm{Chl} \mathrm{mL}^{-1}\right)$ were solubilised with $2 \%(\mathrm{~W} / \mathrm{N}) \beta$-DM and separated in $5-12 \% \mathrm{BN}$ gel gradient followed by SDS PAGE. C, S and M: PSII core, strongly (S) and moderately bound (M) LHCIl trimers; CP: chlorophyll-protein; LHCII-a: CP29 + CP24 + LHCII-t; s: supercomplex; t: trimer d: dimer; m: monomer; Cyt: cytochrome; ATPs: ATP synthase; $\mathrm{CF}_{1}$ : coupling factor 1. Protein standards $(\mathrm{kDa})$ were as follows: bovine serum albumin (66), ovalbumin (45), glyceraldehyde-3-phosphate dehydrogenase (36), carbonic anhydrase (29), trypsinogen (24), trypsin inhibitor (20), a-lactalbumin (14). b Baseline of the densitogram of poplar thylakoid complexes separated by BN PAGE shown in a made by the Phoretix program (adjusted by the user)

Remaining complexes were present as bands containing ATPs complex around PSI and $\mathrm{CF}_{1}$ complex around the PSII monomer band (arrows in Fig. 1a).

\section{General aspects of quantification of thylakoid complexes}

Densitometry is used for determination of the amounts of complexes or polypeptide spots. Here we applied the Phoretix program for the quantitative evaluation of BN and SDS gels. Nevertheless, any densitometry 
applications that enable the quantitative integration of pixel densities on the given band/spot can be also used for evaluation. Comparison of the quantification of bands in Fig. 1a with Phoretix and the open-source ImageJ is given in Additional file 2. To get the real density of bands (calculated as the integrate band volume of the densitogram by the program), the correct determination of the baseline of the densitogram, which is not always a straight line and highly dependent on the structure of the gels, too, is absolutely necessary. Baseline must be adjusted so that those areas of lanes where there are no bands are regarded as zero values (Fig. 1b). Densitometry is based on light absorption by any pigments in the gel that results in dense areas, so we must apply well-defined amounts of Chl (protein) on BN PAGE lanes when Chlprotein complexes are separated. For correct quantitative determinations, band volumes must be directly proportional to the applied amount of protein or Chl. The band volumes and the total volumes per lane [summa] were

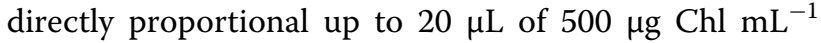
sample application, and the ratio of complexes were also invariable (Fig. $2 \mathrm{a}-\mathrm{c}$ ). Important to remark, bands/spots of the same complexes/proteins can only be compared because of the staining variability of different complexes/ proteins.

Quantitative determination is more complicated when complexes do not separate well and remain together in the same band. This situation may happen among the numerous mega- and supercomplexes, but mainly in the band where PSI move together with PSII-d, as well as in PSII-m band that also includes Cyt $b_{6} f-\mathrm{d}$, and $\mathrm{CF}_{1}$ complexes. In certain cases, even the amount of the Lhc-m band cannot be determined perfectly due to a partial overlap with the Coomassie dye front. One can estimate the amount of PSII-d and PSII-m bands comparing the amount of the $\mathrm{CP} 47$ apoprotein bands (PsbB) in $\mathrm{C}_{2}$ and $\mathrm{C}$ to that of $\mathrm{C}_{2} \mathrm{~S}$ in the denaturing pattern. This latter gives a well separated band in $\mathrm{BN}$ gels. Their ratios in $\mathrm{BN}$ gel should be very similar to those determined in the denaturing gel because the polypeptide pattern and pigment content of these complexes are only slightly different, and Coomassie and pigment stains give the pixel density of
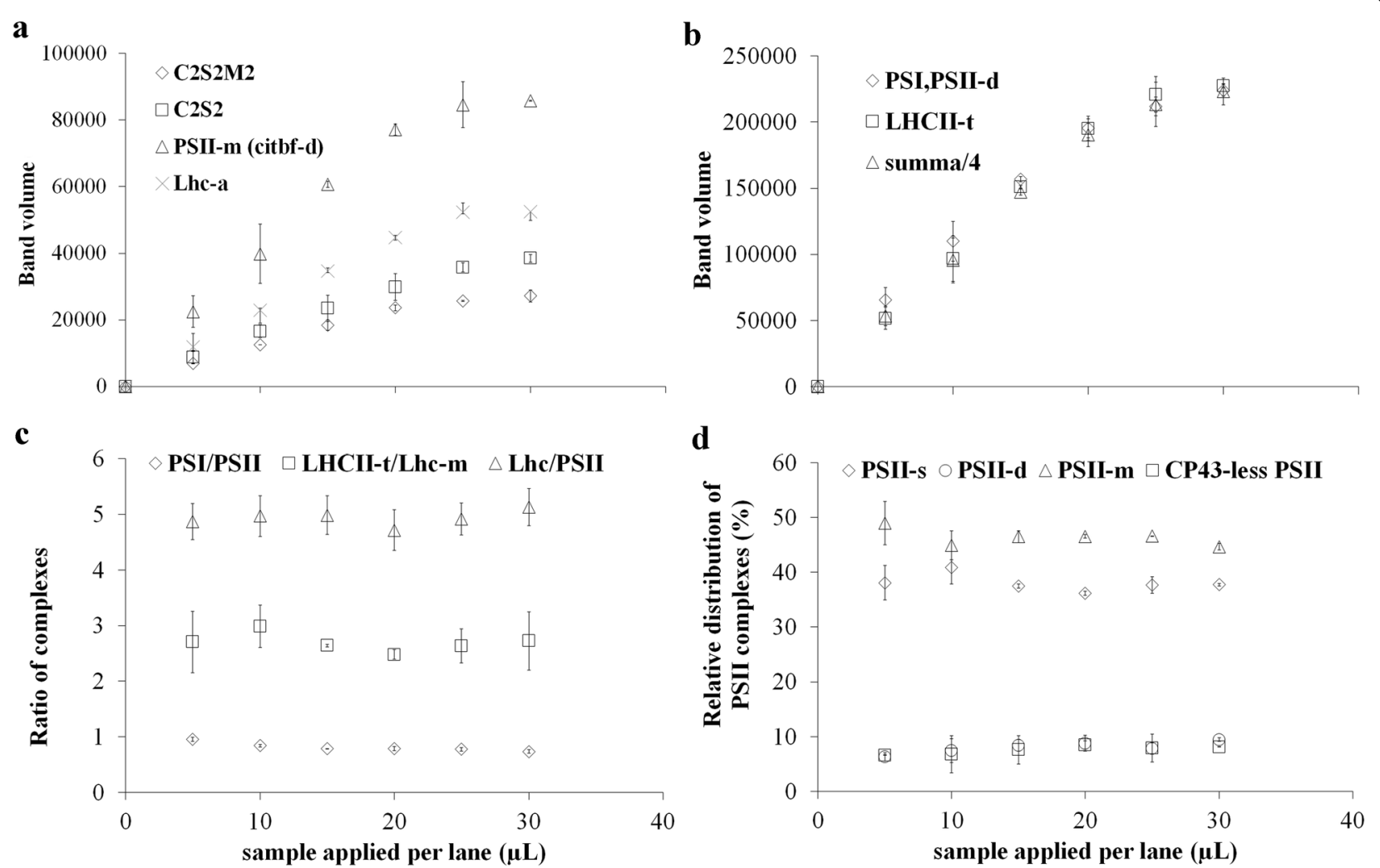

Fig. 2 Proportionality of the thylakoid complexes in BN gels. a, b Band volumes, $\mathbf{c}$ ratios of complexes, and $\mathbf{d}$ distribution of the different PSII forms as the function of the sample volume applied per lane. Complexes are marked as in Fig. 1a, s: supercomplex, d: dimer, m: monomer, summa: total band volumes/lane. Poplar thylakoids (500 $\mathrm{g} \mathrm{Chl} \mathrm{mL}^{-1}$ ) were solubilised using $2 \%(\mathrm{w} / \mathrm{V}) \beta$-DM and separated in 5-12\% gel gradient with three technical repetitions. PSI/PSII and LHCII-t/Lhc-m ratios were calculated from BN bands, LHCII/PSII and distribution of PSII among its different forms are calculated from SDS gel patterns: all Lhcb spots and PsbB spots were taken into account (see Additional file 2) 
bands on BN gels. Similarly, the amount of Lhc-m on the $\mathrm{BN}$ lanes can be calculated using the ratio of its stained polypeptides either to those of LHCII-t or LHCII-a in the denaturing gel. Estimation of the amount of Cyt $b_{6} f-\mathrm{d}$ in PSII- $m$ band that also contain $\mathrm{CF}_{1}$ is a little more complicated. Once the amount of PSII-m is determined, the remaining volumes in the complex band can be roughly distributed between Cyt $b_{\sigma} f-\mathrm{d}$ and $\mathrm{CF}_{1}$ according to the ratio of their apoprotein amounts detected in denaturing gels.

Another challenge in the quantification of thylakoid complexes is that they represent not only different complexes but also different forms of the same type of complex due to solubilisation but even for such physiological reasons that thylakoids are in different developmental or acclimation stages. From the point of view of membrane dynamics, it is important, indeed, to follow the alterations among these forms. Quantities of complexes which do not show fundamental differences in their composition, e.g. similar PSI complexes or Lhc mono/oligomers, can be estimated from the first-dimensional $\mathrm{BN}$ band patterns. However, exact proportion of PSII complexes with different antenna complements cannot be quantified on a similar way. Their proportions can be evaluated from the SDS PAGE pattern using their apoCP47 (PsbB) spot, which is present in all complexes. Basically, it corresponds to the more elegant D1 dot immunoblot method offered by Koochak et al. [54] but can be performed easier. The relative distribution of a complex among its different forms used to characterise changes in the interaction of the different complexes were also invariable in the 5-20 $\mu \mathrm{L}$ range (Fig. $2 \mathrm{~d}$ ). An example of all these calculations is given in Additional file 2: $\mathrm{BN}$-solubilisation, evaluation.

Practical examples of quantitative determinations will be given in chapters characterising large PSI complexes and comparing different thylakoid samples.

\section{Methodological improvements to separate mega-/ supercomplexes in native form}

As a second model, maize thylakoids isolated from untreated and LM treated maize seedlings were used to demonstrate the extraction and resolution of thylakoid complexes, particularly PSI mega-/supercomplexes. In maize leaves, the operation of $\mathrm{C}_{4}$ type photosynthesis requires the collaboration of $\mathrm{M}$ and $\mathrm{BS}$ cells containing granal and agranal chloroplasts, respectively [55]. BS thylakoids contain low amount of PSII complexes. Therefore, they are more convenient for studying large PSI complexes. LM is an inhibitor of prokaryotic type translation [56]. Thus, it strongly interferes with protein synthesis in the chloroplast inhibiting the translation of chloroplast encoded core polypeptides of PSI and PSII but does not affect the accumulation and import of the nuclear encoded Lhc-s [57-59]. The high amount of antennae relative to the core complexes may change the complex interactions.

\section{$\beta$-DM-digitonin detergent mixture gives almost complete solubilisation of maize thylakoids and retain the native state of complexes}

To get a realistic picture about the composition of thylakoids it is important to achieve a complete solubilisation but at the same time to retain the native state of the complexes as far as possible. This mainly depends on the type and concentration of detergent used for solubilisation. The $\beta$-DM and digitonin are the most frequently used detergents in BN PAGE $[3,17]$. A digitonin $-\beta$ $\mathrm{DM} / \alpha-\mathrm{DM}$ mixture was found to be beneficial in sucrose gradient isolation of PSI-LHCII [10] and getting a more stable $\mathrm{C}_{2} \mathrm{~S}_{2} \mathrm{M}_{2}$ supercomplex [60], respectively. Earlier, with Deriphat PAGE, we also got better results with detergent mixtures than using simple detergents in green gels [7]. Therefore, we investigated the efficiency of mild detergents, digitonin and $\beta$-DM used in BN PAGE and for comparison $n$-dodecyl-sucrose (DS) used in Deriphat PAGE [7], together with that of $\beta$-DM/DS-digitonin mixtures for the solubilisation of thylakoids and for the retention of complex interactions.

Concerning the mildness of detergents studied, it was found that supercomplexes, particularly PSI-LHCII, were more stable in the presence of digitonin than using $\beta$ DM or DS (Fig. 3a, b). However, the solubilisation of thylakoids with digitonin only reached about $40-50 \%$ and, in addition, more solubilised material applied to the gel remained at the top of the stacking gel and even about $15 \%$ appeared as a smear near to the start of the separating gel (Fig. 3b). The band pattern showed the retention of the largest PSII supercomplexes and PSI-LHCII complex, CS complex, and an extra, somewhat larger monomer PSII band containing some polypeptides running a little further to the PsbD,A region was also noticeable (Additional file 1: Fig. S3b). Increasing the concentration of digitonin from 1 to $2 \%$ did not cause any difference in the solubilisation efficiency and band pattern in agreement with the fact that digitonin was found to solubilise only the stroma and grana margin lamellae [22].

While the use of digitonin only resulted in a partial solubilisation, $\beta$-DM was able to solubilise the maize $\mathrm{M}$ thylakoids almost completely $(>95 \%)$ even at low $(0.5 \%)$ concentration. However, there was some discrepancy using $0.5 \% \beta$-DM: the amount of Lhc-m was always elevated compared to higher $\beta$-DM concentrations. The PSI-LHCII supercomplex was very sensitive to $\beta$-DM. In addition, the elevation of $\beta$-DM concentration from 0.5 to $2 \%$ led to a shift from larger PSII supercomplexes 


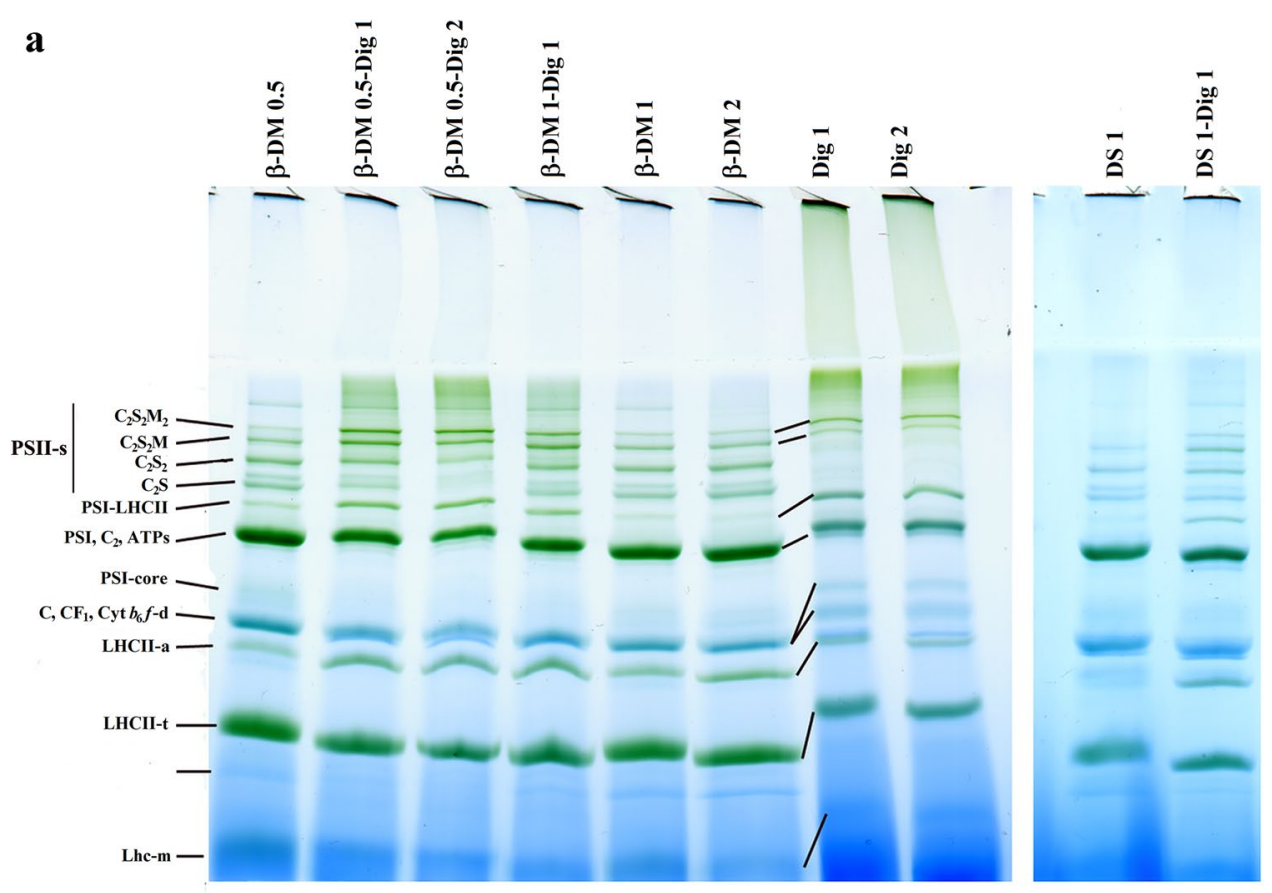

$\mathbf{b}$

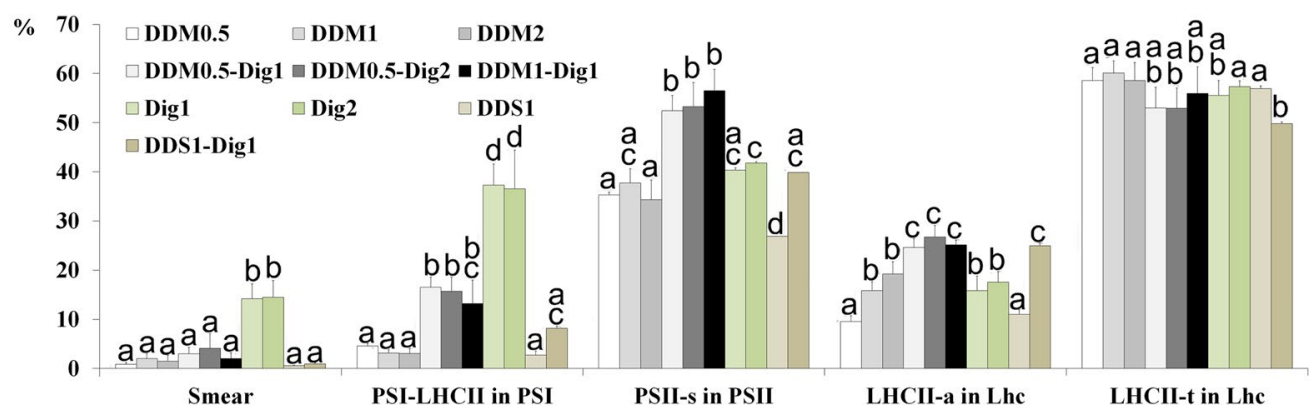

Fig. 3 Effect of solubilisation protocols on the complex pattern of maize mesophyll thylakoids. a Representative gel patterns of thylakoids $\left(500 \mu \mathrm{g} \mathrm{Chl} \mathrm{mL}{ }^{-1}\right)$ solubilised using different detergents and detergent mixtures (given in \% w/N), and separated in 4.3-12\% gel gradient. Dig: digitonin, $\beta$-DM: $n$-dodecyl- $\beta$-D-maltoside, DS: $n$-dodecyl-sucrose. b The ratio of the more sensitive bands is given in \% of the total PSI, PSII and Lhc amounts, respectively. Complexes are marked as in Fig. 1a. Smear is the bluish green staining at the beginning of the separating gel where bands cannot be noticed clearly given in \% of total stain in the lane. Effects of different detergents were compared using one-way ANOVA with Tukey's multiple comparison test for each group separately $[P<0.05 ; 6$ technical repetitions: $3 \times$ solubilisation and separation in $2-2$ lanes]. Significant differences are indicated by different letters in each group separately

to smaller ones. A mixture of $\beta$-DM and digitonin gave better results: it also solubilised thylakoids similarly to $\beta$-DM alone, the large supercomplexes and LHCII-a were retained, PSI-LHCII being the most sensitive but still being retained much better than using only $\beta$-DM for solubilisation, in agreement with the findings in [10]. LHCII-t was hardly sensitive to the presence of detergents as it was also found earlier [24]. DS proved to be a little harsher than $\beta$-DM, but also became milder in a mixture with digitonin. It follows, if we can find milder detergents than $\beta$-DM with good solubilisation ability (an example may be $\alpha$-DM mentioned by Pagliano et al. [61]), and use it together with digitonin, it may be more useful to preserve the native state of complexes.

The best resolution of complexes in thylakoids isolated

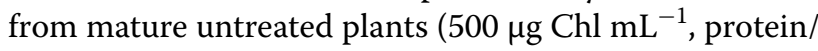
Chl ratio around 4-5) was obtained with $1 \% \beta$-DM and $1 \%$ digitonin, and the retention of complexes was not worse than using mixtures of lower $\beta$-DM concentration (Fig. 3b). Thus, we used this detergent mixture in solubilisation of mature thylakoids. In the case of still developing thylakoids, however, one must take into consideration 
the high protein content of the samples, too. Lowering the Chl concentration to $250 \mu \mathrm{g} \mathrm{mL}^{-1}$ during solubilisation usually gave satisfactory results if the protein per $\mathrm{Chl}$ ratio doubled (e.g. after $16 \mathrm{~h}$ greening of etiolated plant material). Checking the protein/Chl ratio is also advised using thylakoids isolated from plants after strong stresses [28].

\section{A 4.3-8\% BN gel system is beneficial for the separation of the large complexes}

Earlier, 5-12\% BN gradient gels were used for the separation of thylakoid complexes (e.g. [18, 20, 28]). Later on, higher resolution of megacomplexes was achieved either using gel gradients of $4 \%$ starting concentration [34, 37] or so-called large-pore gels ([16], and others working in the same laboratory). Resolution of PSII supercomplexes on $\mathrm{CN}$ gels could have been improved by decreasing the end concentration of the separating gel [62]. In our practice, though the resolution of mega-/supercomplexes has already been promoted by the decrease in the starting acrylamide concentration to $4.3 \%$, it was significantly further facilitated by using $4.3-8 \%$ gradient gels (Fig. 4). It allowed large complexes to run further into the gel resulting in higher resolution of the bands in the PSII-m and (PSI-NDH-mc) region, while the resolution of bands larger than PSI-NDH-mc was perhaps slightly lower than in large-pore gels [16]. In addition, the presence of glycerol in all parts of the gel contributed to the preservation of the native state of complexes (even in denaturing gels-see Additional file 1: Fig. S1b).

Henceforth, $4.3-12 \%$ gels were used aiming at the separation of all thylakoid complexes in 1st and 2nd dimension BN/CN PAGE, but we employed $4.3-8 \%$ gels to obtain better separation of complexes in the mega-/ supercomplex region.

\section{Example 1: Characterisation of large PSI complexes}

While in maize $\mathrm{M}$ thylakoids PSII supercomplexes dominated the BN gel region above the PSI band, mainly PSI-mc bands could be seen in BS thylakoids (Fig. 5). In BS thylakoids, four PSI-mc bands (PSI-mc1-4) were detected running above the PSI-LHCII band together with some faint bands (seemingly also PSI-mc-s). The molecular mass of PSI-mc1-4 complexes calculated from the calibration curve given in Additional file 1: Fig. S4 using internal standards including the molecular masses determined in $[9,63]$ were about 1740, 1325, 1140, and $935 \mathrm{kDa}$, respectively. Though PSII-m was the main PSII form in the BS thylakoids, large PSII supercomplexes and PSII-d, supposed to function in redox poise control of cyclic electron transport [64], could be also detected even in the cleanest BS preparations. The band- and polypeptide patterns of the complexes separated by $\mathrm{BN}$ or $\mathrm{CN}$ PAGE were practically identical (Additional file 1: Fig. S5) as it was also demonstrated by Järvi et al. [16] in Arabidopsis plants.

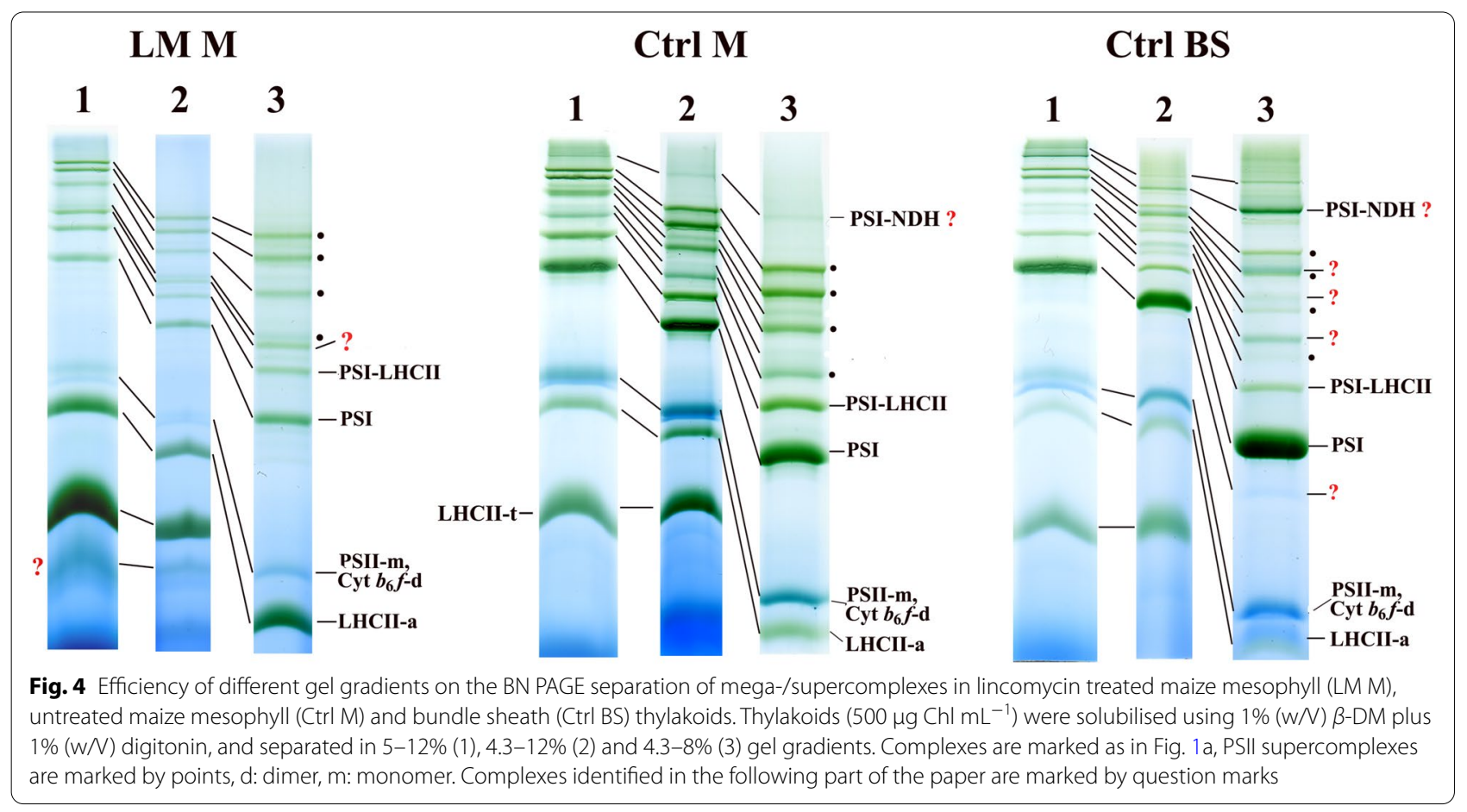




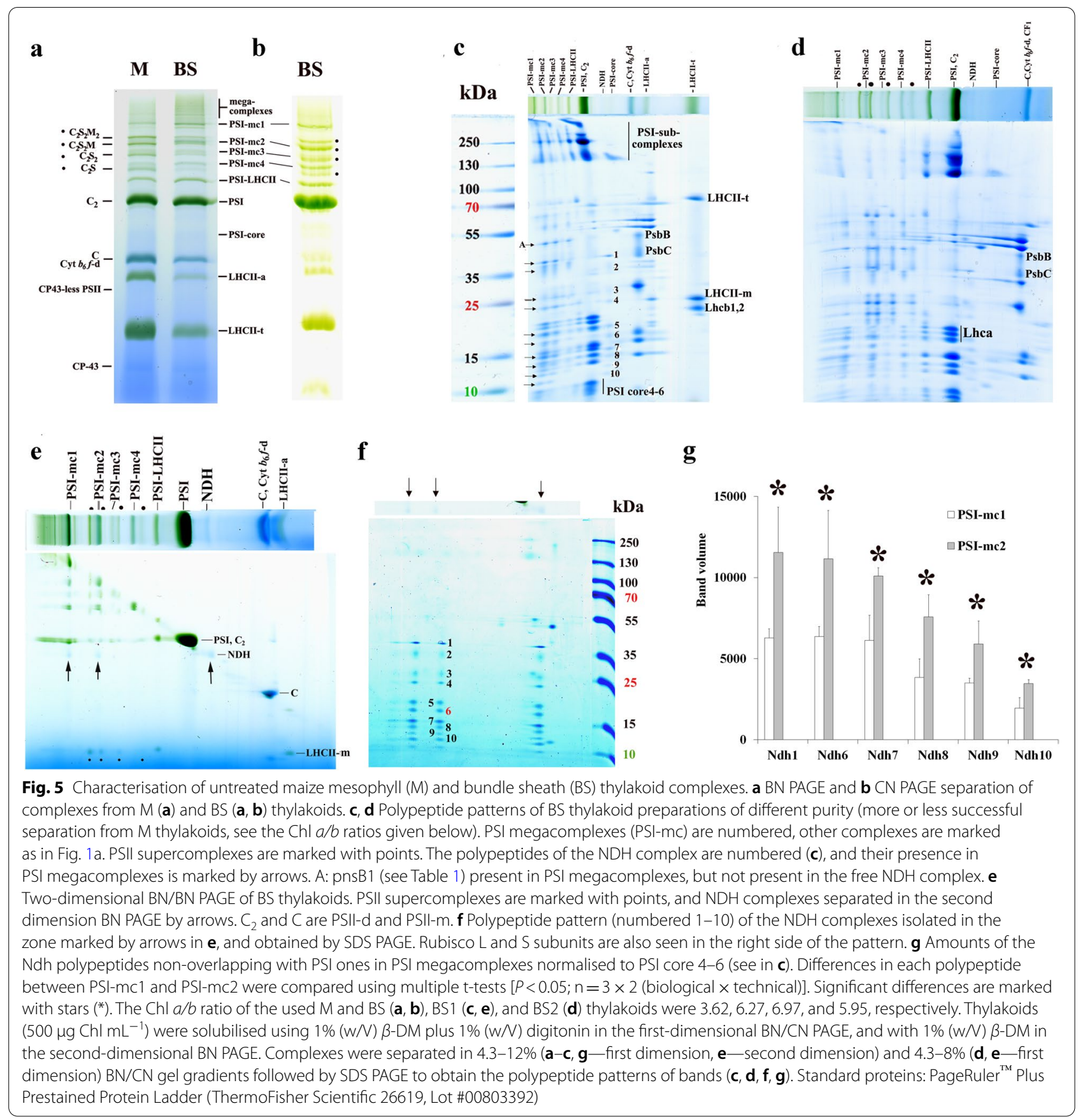

Using higher resolution $\mathrm{BN}$ gels [4.3-8\% gradient], the PSI mc1-4 bands were separated from PSII-s bands. It was clearly visible in the less clean BS samples of relatively lower $\mathrm{Chl} a / b$ ratio (Fig. $5 \mathrm{~d}$ ). In Arabidopsis thylakoids solubilised with digitonin, $\mathrm{BN}$ bands around the position of PSI-mc1 and PSI-mc3 were supposed to be trimeric and dimeric forms of PSI, respectively, and were interpreted as solubilisation artefacts [38]. Later, the PSImc1-like band was identified as PSI-NDH-mc [16, 49,
63]. In the more complex first-dimensional BN pattern of maize BS thylakoids, a $525 \mathrm{kDa}$ band appeared migrating below the main PSI band (polypeptide components are marked with numbers in Fig. 5c) which corresponded to the NDH complex of $\sim 550 \mathrm{kDa}$ [65-67]. However, subcomplex B of the NDH complex could be hardly detected in the polypeptide pattern of this solubilised complex (Table 1). This complex was shown to accumulate preferentially in BS thylakoids $[68,69]$. According to the 
Table 1 Polypeptides detected in the PSI-mc-s and the NDH complex by MS analysis

\begin{tabular}{|c|c|c|c|c|c|c|c|c|}
\hline & Plant & Protein & $\begin{array}{l}\text { M.W } \\
\text { Da }\end{array}$ & $\begin{array}{l}\text { PSI-mc1 } \\
\text { Score }\end{array}$ & $\begin{array}{l}\text { PSI-mc2 } \\
\text { Score }\end{array}$ & $\begin{array}{l}\text { PSI-mc3 } \\
\text { Score }\end{array}$ & $\begin{array}{l}\text { PSI-mc4 } \\
\text { Score }\end{array}$ & $\begin{array}{l}\text { NDH } \\
\text { Score }\end{array}$ \\
\hline \multicolumn{9}{|l|}{ PSI } \\
\hline A0A3B6UD72 & Z. $m$ & psaA & 83,055 & $4357^{x, a}$ & $4362^{x}$ & $1862^{\mathrm{a}, \mathrm{x}}$ & $1568^{x}$ & \\
\hline A0A5P8KLV9 & Z. $m$ & psaB & 82,517 & $5909^{x, a}$ & $4607^{x}$ & $3917^{a, x}$ & $1866^{x}$ & \\
\hline A0A5P8KLH3 & Z. $m$ & $\mathrm{psaC}$ & 8893 & $15414^{x, a}$ & $5503^{x}$ & $6691^{\mathrm{a}}$ & $6269^{x}$ & \\
\hline A0A317YJL6 & Z. $m$ & psaD & 21,563 & $41944^{x, y}$ & & $30676^{b, a, x, y}$ & & \\
\hline B4FAW3 & Z. $m$ & psaD & 21,563 & & $57637^{y, x}$ & & $18722^{x}$ & \\
\hline A0A3L6E4J8 & Z. $m$ & psaE & 14,427 & $32357^{x, y}$ & $28888^{y, x}$ & $27,812^{b, a, x, y}$ & $4825^{x, a, b}$ & \\
\hline A0A096RAVO & Z. $m$ & $\mathrm{psaF}$ & 24,487 & $30393^{y, a}$ & $42839^{y, x}$ & $30,393 b, a, x, y$ & $6960^{x, y}$ & \\
\hline B6U534 & Z. $m$ & PsaG & 15,304 & $6320^{x, a}$ & $5675^{x}$ & $5733^{a, x}$ & $1368^{x}$ & \\
\hline A0A3L6E9J9 & Z. $m$ & psaH & 14,920 & $11956^{x}$ & $8266^{x}$ & $20186^{b, x, y}$ & $756^{\mathrm{a}}$ & \\
\hline A0A3L6E041 & Z. $m$ & psak & 13,595 & $3790^{x}$ & $3444^{x}$ & $3790^{x, a}$ & $3697^{x}$ & \\
\hline A0A3L6DL54 & Z. $m$ & psaL & 22,240 & $985^{x}$ & $829^{x}$ & $1183^{a}$ & $2609^{x}$ & \\
\hline A0A3L6FDJ3 & Z. $m$ & psan & 23,369 & $3461^{x}$ & & $2576^{\mathrm{a}}$ & & \\
\hline A0A3L6F840 & Z. $m$ & Ihca-P4 & 29,477 & $11070^{x, a, b}$ & $9737^{x}$ & $10327^{a, x, y}$ & & \\
\hline A0A3L6EJZ9 & Z. $m$ & Ihca5 & 33,854 & $376^{a}$ & & & & \\
\hline AOAOF7H1K9 & H.b & Ihca6 & 29,515 & & $1057^{y}$ & & & \\
\hline A0A6M3WH11 & C.p & Ihca6 & 28,442 & & $1018^{y}$ & & & \\
\hline \multicolumn{9}{|l|}{ NDH chloroplastic } \\
\hline \multicolumn{9}{|l|}{ e-donor binding } \\
\hline K7TFI4 & Z. $m$ & ndhU & 25,323 & $194^{\mathrm{a}}$ & $7106^{x}$ & & & $3688^{x}$ \\
\hline A0A3L6GCK8 & Z. $m$ & ndhu & 25,309 & $7627^{x}$ & & & & \\
\hline \multicolumn{9}{|l|}{ Subcomplex A } \\
\hline A0A5P8KLE9 & Z. $m$ & ndhH & 45,629 & $12097^{x, a}$ & $12285^{x}$ & $479^{a}$ & & $7474^{x}$ \\
\hline A0A5P8KLE6 & Z. $m$ & ndhl & 21,143 & $27850^{x, a}$ & $13488^{x}$ & & & $15901^{x}$ \\
\hline A0A5P8KLA0 & Z. $m$ & ndhJ & 18,657 & $7105^{x, a}$ & $26589^{x}$ & & & $18907^{x}$ \\
\hline A0A5P8KLC5 & Z. $m$ & ndhK & 27,902 & $11795^{x}$ & $7443^{x}$ & & & $5595^{\times}$ \\
\hline B4FRX4 & Z. $m$ & ndhM & 23,964 & $17739^{x}$ & $11325^{x}$ & & & $6239^{x}$ \\
\hline A0A3L6FA99 & Z. $m$ & ndhN & 23,024 & $2640^{x, a}$ & $17139^{x}$ & & & $9570^{x}$ \\
\hline A0A3L6DTN1 & Z. $m$ & ndhO & 16,595 & $12097^{x, a}$ & $3167^{x}$ & & & $3296^{x}$ \\
\hline \multicolumn{9}{|l|}{ Subcomplex B } \\
\hline A0A3L6GBC8 & Z. $m$ & pnsB1 & 51,403 & $15044^{y, x, a, b}$ & $13418^{x, y}$ & $4167^{a, b, x, y}$ & $4529^{x, a, b, y}$ & \\
\hline A0A3L6DA24 & Z. $m$ & pnsB2 & 37,902 & $32626^{x, a, b, y}$ & $23464^{y}$ & $8554^{a, b, x, y}$ & $2260^{b, a}$ & \\
\hline B4FWG2 & Z. $m$ & pnsB2 & 37,902 & & $27745^{x}$ & & $15228^{x}$ & \\
\hline A0A3L6E2Y7 & Z. $m$ & pnsB3 & 20,798 & $4649^{x, y}$ & $5414^{x}$ & & & \\
\hline A0A1D6HK95 & Z. $m$ & pnsB4 & 31,198 & & & $1985^{x}$ & & \\
\hline A0A1D6HK96 & Z. $m$ & ndhB4 & 20,603 & $2637^{y}$ & & $2637^{b}$ & & \\
\hline A0A3L6FDN2 & Z. $m$ & pnsB5 & 25,605 & $10998^{y, x}$ & $10306^{x}$ & $1864^{\mathrm{a}, \mathrm{x}, \mathrm{y}}$ & $7131^{x, a, b}$ & \\
\hline \multicolumn{9}{|c|}{ Membrane subcomplex } \\
\hline A0A3B6UD78 & Z. $m$ & ndhA & 40,346 & $579^{a}$ & & $909^{\mathrm{a}}$ & & \\
\hline P25706 & Z. $m$ & ndhA & 40,485 & $5094^{x}$ & $6331^{x}$ & & & $5854^{x}$ \\
\hline A0A5P8KL97 & Z. $m$ & ndhc & 13,851 & $1596^{x}$ & $1606^{x}$ & & & \\
\hline A0A5P8KM09 & Z. $m$ & ndhD & 56,316 & $3444^{x, a}$ & $4815^{x}$ & $503^{x, a}$ & $985^{x}$ & \\
\hline A0A5P8KLF2 & Z. $m$ & ndhF & 82,950 & $5788^{x, a}$ & $6092^{x}$ & $2561^{a, x}$ & $2077^{x, a}$ & \\
\hline
\end{tabular}


Table 1 (continued)

\begin{tabular}{|c|c|c|c|c|c|c|c|c|}
\hline & Plant & Protein & $\begin{array}{l}\text { M.W } \\
\text { Da }\end{array}$ & $\begin{array}{l}\text { PSI-mc1 } \\
\text { Score }\end{array}$ & $\begin{array}{l}\text { PSI-mc2 } \\
\text { Score }\end{array}$ & $\begin{array}{l}\text { PSI-mc3 } \\
\text { Score }\end{array}$ & $\begin{array}{l}\text { PSI-mc4 } \\
\text { Score }\end{array}$ & $\begin{array}{l}\text { NDH } \\
\text { Score }\end{array}$ \\
\hline \multicolumn{9}{|c|}{ Lumenal subcomplex } \\
\hline A0A1D6JYG6 & Z. $m$ & pnsL1 & 25,683 & $10829^{y, a, b, x}$ & $10571^{x, y}$ & $5036^{\mathrm{b}, \mathrm{a}, \mathrm{x}}$ & & $6759^{x, y}$ \\
\hline A0A1D6HBD7 & Z. $m$ & pnsL2 & 24,200 & $27466^{y, a, b, x}$ & $20585^{x, y}$ & $4104^{b, a, x, y}$ & & $15443^{x, y}$ \\
\hline A0A3L6E7E6 & Z. $m$ & pnsL3 & 25,686 & $5953^{y}$ & $5382^{x}$ & $2365^{b, a}$ & $1008^{\mathrm{a}}$ & \\
\hline A0A1D6HQR2 & Z. $m$ & pnsL3 & 28,290 & & $5957^{y}$ & & $879^{b}$ & \\
\hline
\end{tabular}

Data were obtained from bands originating from two different isolations and separations. Analysis was performed in maize (mark $a$ ) and PSI-NDH (mark $b$ ) databases (isolation 1, separation in 4.3-12\% BN gel gradient) and maize (mark $x$ ) and PSI-NDH (mark y) databases (isolation 2, separation in 4.3-8\% BN gel gradient) acquired form UniProtKB. Search results can be found in Additional file 3: MS results 1 (isolation 1) and Additional file 4: MS results 2 (isolation 2). Superscripts show the hits. The highest scores (first superscript marks) are shown. Nomenclature of Shikanai [72] was used in the case of chloroplast NDH. The pnsB1 present in PSI-mc-s but not in the free NDH complex and ndhH corresponds to band $\mathrm{A}$ and band 1, respectively, in Fig. $5 \mathrm{C}$

CA: carbonic anhydrase; C.p.: Carica papaya; FS: iron-sulphur protein; FV: flavoprotein; H.b.: Hypseocharis bilobata; su: subunit; Z.m.: Zea mays

polypeptide patterns obtained by the second-dimensional BN PAGE followed by SDS PAGE, this complex could be isolated with similar polypeptide pattern and ratios in the free complex, PSI-mc1, and PSI-mc2, and in a larger megacomplex, too (Fig. 5f, Additional file 1: Fig. S6).

Comparing the amounts of polypeptides in the different PSI complexes normalised to PSI core bands, the band volumes and ratios of PSI polypeptides were similar in PSI, PSI-LHCII and PSI-mc1,2,4 (Additional file 1: Fig. S7). However, using the same normalisation method elevated amount of Ndh polypeptides were found in PSImc2 compared to PSI-mc1 (Fig. 5g). According to the molecular mass of PSI $(\sim 580 \mathrm{kDa})$ and NDH $(\sim 550 \mathrm{kDa})$, the PSI-mc1 and PSI-mc2 may represent PSI/NDH ratio of two and one, respectively. PSI megacomplexes with different PSI/NDH ratio of two and more were also detected earlier [34, 37, 43, 48, 49, 70, 71]. In agreement with above mentioned data, the relationship of PSImc1,2,4 bands obtained by the second-dimensional BN PAGE verified that the solubilisation products of PSImc1 were PSI-mc1,2,4, and NDH complex and those of PSI-mc2 PSI-mc2,4 and NDH complex (Fig. 5e). To the contrary, no NDH spot was observed in the pattern of PSI-mc4. A smaller NDH related spot (NDH part) originating from this megacomplex can be suspected on the stained second-dimensional BN pattern of this complex (Additional file 1: Fig. S8). The polypeptide composition of the PSI-mc1,2,4 determined by mass spectrometry supported their PSI-NDH complex characteristics (Table 1). While PSI-mc1,2 contained all the main components of the NDH complex, the polypeptides of subcomplex A apparently were not present in PSI-mc4. PSI-mc2 also contained PSII polypeptides due to its close running to $\mathrm{C}_{2} \mathrm{~S}_{2} \mathrm{M}$ (see Additional files 3 and 4).

The polypeptide composition of the PSI-mc3 band, appearing in low amount, was not as clear as that of other PSI megacomplexes. Nevertheless, many polypeptide

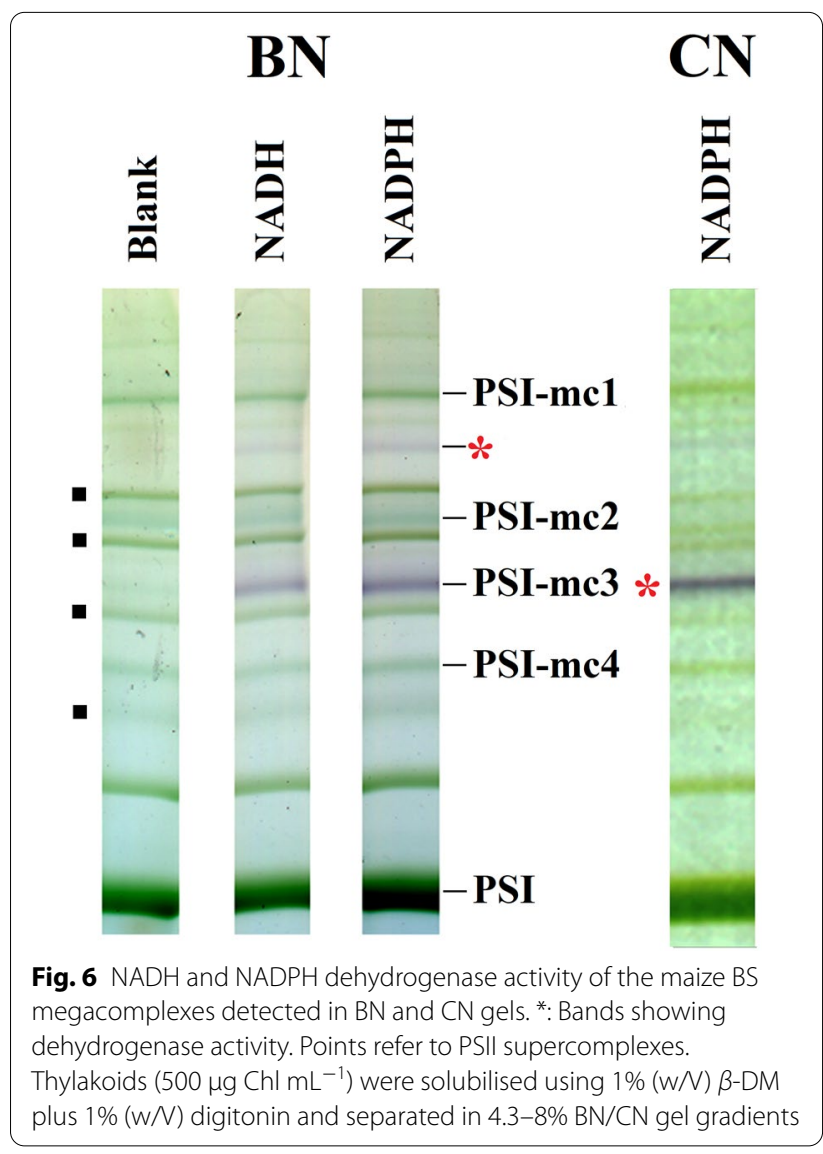

components of PSI and chloroplast NDH were present (Table 1). PSI-mc3 also contained PSII polypeptides due to its close running to $\mathrm{C}_{2} \mathrm{~S}_{2}$. In chloroplasts, PSI$\mathrm{NDH}$ megacomplexes are known to utilise ferredoxin as reductant [72-74] bound to the complex by the NdhS protein that shares structural similarity with PsaE [75]. As subcomplex A was lacking and electron donor binding polypeptides were not detected in PSI-mc3 (Table 1), and 
Table 2 Mitochondrial complex I components found in PSI-mc3

\begin{tabular}{|c|c|c|c|c|}
\hline Accession & Plant & Protein & $\begin{array}{l}\text { M.W } \\
\text { Da }\end{array}$ & $\begin{array}{l}\text { PSI-mc3 } \\
\text { score }\end{array}$ \\
\hline \multicolumn{5}{|c|}{ Mitochondrial complex I } \\
\hline A0A1D6L210 & Z.m & FS1 & 80,666 & $22069^{a, x}$ \\
\hline B4FGH7 & Z.m & FS4 & 17,250 & $1834^{\mathrm{a}}$ \\
\hline A0A1D6NDN3 & Z.m & FS7 & 13,933 & $3128^{a, x}$ \\
\hline A0A3L6G2J2 & Z.m & $\mathrm{FV} 1$ & 55,160 & $6878^{a}$ \\
\hline B6T6U3 & Z.m & $\mathrm{FV} 1$ & 55,146 & $1864^{x}$ \\
\hline B4FPX5 & Z.m & $\mathrm{FV} 2$ & 35,177 & $1867^{a}$ \\
\hline Q6R9J9 & Z.m & nad2 & 53,634 & $1035^{\mathrm{a}}$ \\
\hline Q6R9H4 & Z.m & nad5 & 74,440 & $2815^{\mathrm{a}}$ \\
\hline Q5K097 & Z.m & nad9 & 22,916 & $12347^{a, x}$ \\
\hline K7USN8 & Z.m & CA1 & 28,401 & $549^{a}$ \\
\hline A0A3L6DZ16 & Z.m & CA2 & 29,625 & $3062^{a, x}$ \\
\hline A0A3L6DF13 & Z.m & alpha5 & 24,897 & $1701^{a}$ \\
\hline A0A317YH98 & Z.m & alpha6 & 15,131 & $396^{a}$ \\
\hline A0A1D6HPC4 & Z.m & alpha9 & 37,931 & $6035^{a, x}$ \\
\hline B4FCV1 & Z.m & alpha12 & 18,523 & $3914^{a}$ \\
\hline A0A317YFY5 & Z.m & alpha13 & 15,963 & $5430^{a}$ \\
\hline A0A317Y753 & Z.m & beta10 & 12,493 & $2733^{a}$ \\
\hline B6TLXO & Z.m & 10.5 kDa su & 11,094 & $4786^{a}$ \\
\hline A0A1D6GG96 & Z.m & $23 \mathrm{kDa}$ su & 25,650 & $8236^{a}$ \\
\hline B6TMG9 & Z.m & $29 \mathrm{kDa} s u$ & 27,872 & $1448^{\mathrm{a}}$ \\
\hline
\end{tabular}

Separations, MS analyses, and presentation were done as in Table 1 enzymes of similar size with $\mathrm{NAD}(\mathrm{P}) \mathrm{H}$ dehydrogenase activity were discovered in chloroplasts of rice (Oryza sativa) flag leaves and panicles [69], we investigated $\mathrm{NAD}(\mathrm{P}) \mathrm{H}$ dehydrogenase activity of megacomplexes. Among the main PSI megacomplexes, only PSI-mc3 band showed high dehydrogenase activity (Fig. 6). The detected activity was similar with either NADH or NADPH. Faint activity could be also seen in a band of larger molecular mass running between PSI-mc1 and $\mathrm{C}_{2} \mathrm{~S}_{2} \mathrm{M}_{2}$ complex. Activity-stained complexes were the same in $\mathrm{BN}$ and $\mathrm{CN}$ gels. $\mathrm{NAD}(\mathrm{P}) \mathrm{H}$ dehydrogenase activity of the PSI-mc3 band may come from contaminating mitochondrial complex I ( 1000 kDa) [76] since many polypeptide components of mitochondrial NADH dehydrogenase [77] were also detected (Table 2). However, $\mathrm{NAD}(\mathrm{P}) \mathrm{H}$-dependent $\mathrm{NDH}$ complexes of similar size were detected in rice [78], and a dimeric NDH related complex of $\sim 1000 \mathrm{kDa}$ was also found in BS chloroplasts functioning in the carbon concentration mechanism [67]. PSI dimers of similar size were also detected in BN PAGE [38] and by single particle analysis [43]. Interestingly, Proton Gradient Regulation 5 (PGR5) was also detected in PSI-mc3 (see Additional file 3). Different complexes of similar molecular mass present in the same band cannot be excluded in BN PAGE separations. The detailed composition of this band needs further examination.

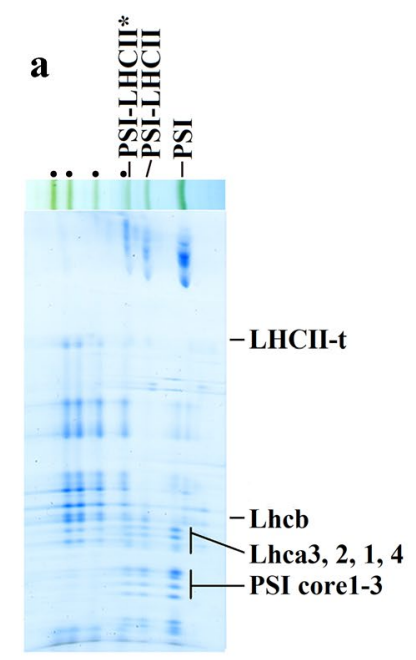

b

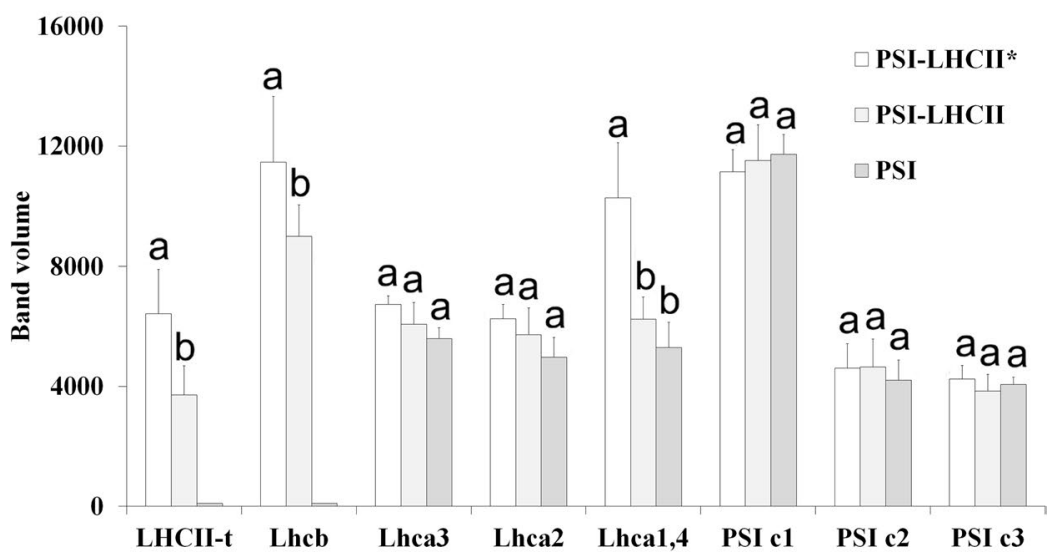

Fig. 7 Polypeptide patterns of PSI-LHCII supercomplexes separated from mesophyll thylakoids of lincomycin treated maize. a BN/SDS gel pattern of thylakoids. Points refer to PSIl supercomplexes. Thylakoids $\left(500 \mu \mathrm{g} \mathrm{Chl} \mathrm{mL}{ }^{-1}\right)$ were solubilised using 1\% (w/V) $\beta$-DM plus $1 \%$ (w/N) digitonin, and complexes were separated in 4.3-8\% gel gradients followed by SDS PAGE. PSI-LHCII*: large PSI-LHCII band. b Comparison of the polypeptide profiles of PSI and PSI-LHCII supercomplexes normalised to PSI core 1-3 (see in $\mathbf{a}$. Variations in the amounts of polypeptides in the different complexes were compared using two-way ANOVA with Tukey's multiple comparison test $[P<0.05 ; n=2 \times 3$ (biological $\times$ technical)]. Significant differences are indicated separately in each polypeptide/LHCII-t group by different letters 
In maize $M$ thylakoids isolated from LM treated plants another large PSI complex $(\sim 825 \mathrm{kDa})$ was discovered by two-dimensional BN/SDS PAGE (indicated by asterisk in Fig. 7a) which was sometimes also observed in the untreated control ones ( $c f$. Additional file 1: Fig. S9a). The polypeptide composition of the new PSI-s differed from that of the PSI-mc bands detected in BS thylakoids, the former being more like to that of PSI-LHCII. The PSILHCII band contains one copy of all Lhca1-4 complexes and an LHCII trimer [39]. There are literature data on larger PSI complexes binding more LHCII trimers [23, 42-45]. However, small differences in the LHCII-t and Lhcb content between PSI-LHCII and PSI-LHCII* may be rather due to the partial overlap of the latter with the $\mathrm{C}_{2} \mathrm{~S}$ complex. Otherwise, PSI-LHCII* showed totally similar composition and position in the separation pattern to the PSI-core-6Lhca-LHCII complex isolated from Arabidopsis, which binds an extra Lhca1,4 dimer, and was found more abundant in State 2 [79]. Indeed, the Lhca1,4 content was also higher in PSI-LHCII* complex (Fig. 7b).

Summing up the results about the composition of large-mass PSI bands in maize thylakoids, complexes proved to be differently solubilised PSI-NDH megacomplexes in which PSI binds either total NDH (PSI-mc1,2) or a part of it (PSI-mc4) or PSI complexes with different antenna complements (PSI-LHCII, PSI-LHCII*). To clarify the nature of the PSI-mc3 band needs better resolution of this gel region.

\section{Example 2: Quantitative comparison of complexes in different thylakoid samples}

From the quantitative point of view, we may compare the changes in the amounts and interactions of thylakoid complexes in the different samples. Before comparison, the sum band volume of all BN lanes must be normalised to the same value because they may differ due to subtle differences in solubilisation or pipetting, see also in Fagioni et al. [27]. If the analysis is free of these problems, then lanes of the same Chl content isolated from mature plants, having a protein/Chl ratio of $4.19 \pm 0.46$, gave very similar summa volumes [28].

Depending on our interest/purpose, the changes in the amounts of complexes can be demonstrated in multiple ways. One possibility is to display their absolute amount, i.e. how the $\mathrm{Chl}$ content present in $\mathrm{g}$ fresh or dry weight, per $\mathrm{cm}^{2}$ leaf area, in the whole leaf or plant is distributed among the complexes. It means that the summa band volume of the lane corresponds to the Chl concentration or Chl content of the leaf or plant which is divided among the complexes according to their ratios. It seems to be more suitable to use CN PAGE for this purpose because of the different $\mathrm{Chl}$ per protein ratios of complex bands. Interestingly, however, there were no large differences between the band volumes of complexes obtained by $\mathrm{CN}$ and BN PAGE (Additional file 1: Fig. S10) except in the case of PSII-m, the amount of which at least doubled in the BN PAGE pattern due to its low $\mathrm{Chl} /$ protein content and the presence of other complexes (Cyt $b_{6} f-\mathrm{d}$, $\mathrm{CF}_{1}$ ). However, this presentation is influenced not only by changes in the distribution of complexes but also by other parameters such as the fresh or dry weight or area differences or growth characteristics of the leaf or plant.

To get a clear picture on alteration of the composition or complex interactions in thylakoids isolated from multiple or differently treated plants, it is better to compare either the amount of similar complexes or their percentage of a given sample (e.g. control) in samples normalised to the same total volume per lane. This latter plotting enables a better distinction of variations in the thylakoid composition. The ratio of complexes and the distribution among the different forms of a complex may be also important. However, it must be kept in mind that the solubilisation conditions of thylakoid being in different developmental/acclimation stage may not be the same, and varying the detergent concentration also causes changes in the complex interactions.

As an example, the amount of the complexes and the complex interactions of $M$ thylakoids of LM treated maize plants were compared to those of controls. The Chl content of treated leaves decreased only moderately to about $73 \%$ of the control (from $1648 \pm 140$ to $\left.1205 \pm 235 \mu \mathrm{g} \mathrm{Chl} \mathrm{g}^{-1} \mathrm{FW}\right)$. However, the Chl $a / b$ ratio was strongly lowered: from $4.03 \pm 0.04$ and $3.51 \pm 0.12$ in the controls to $2.90 \pm 0.22$ and $2.56 \pm 0.22$ in LM treated leaves and $M$ thylakoids, respectively. In agreement with these data, granum structures (Additional file 1: Fig. S11, see also in $[80,81])$ and light-harvesting complexes were much more abundant in $\mathrm{M}$ chloroplasts of LM treated, than in untreated control plants (Fig. 8a, b), and even free Lhca dimers were present (Fig. 8a, b, d, see also Additional file 1: Fig. S12). Interestingly, a complex of similar polypeptide pattern to LHCII-a running a little further than CS (probably LHCII-a-d) was also observed in LM treated thylakoids, which may originate from PSII megacomplexes in which $\mathrm{M}$-trimers are interacting [62]. The ratio of complexes active in the electron transport (PSI, PSII, Cyt $b_{6} f$ ) significantly decreased, while that of the antennae increased compared to control (Fig. 9a) leading to an unchanged PSI to PSII, and elevated antennae per PS-core ratios (Fig. 9b). The shift of PSII forms to PSII supercomplexes and the higher PSI-LHCII/PSI and Lhc-m/LHCII-t ratio (Fig. 9b, c) may be related to the maintenance some photosynthetic activity and/or operation of different protective mechanisms in $M$ thylakoids of LM treated plants [33] which may sustain the viability of plant. 


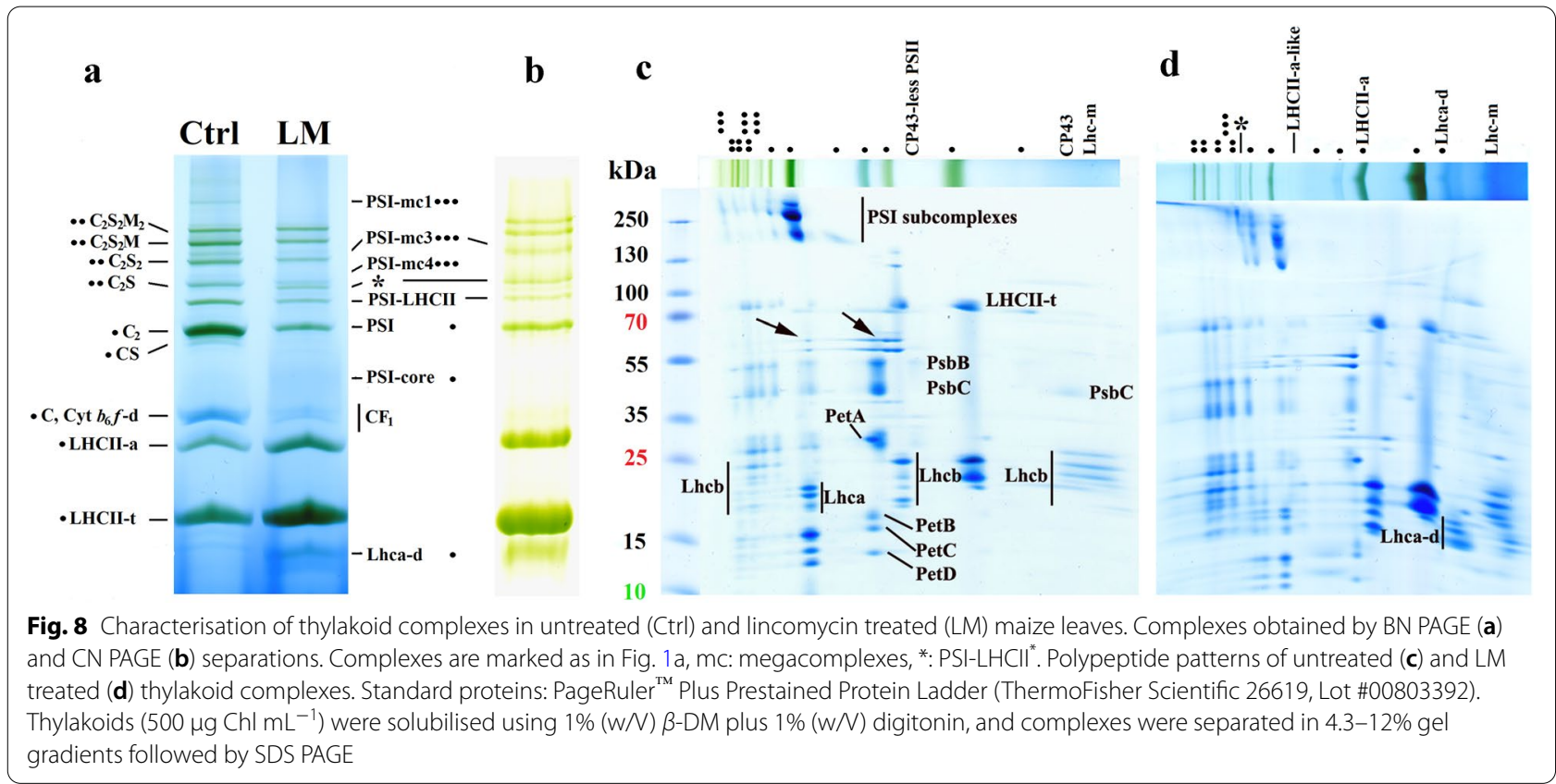

\section{Conclusions}

Solubilisation of thylakoids with $1 \%(\mathrm{w} / \mathrm{V}) \beta$-DM plus $1 \%$ $(\mathrm{w} / \mathrm{V})$ digitonin and increasing the resolution of the large complexes using $4.3-8 \% \mathrm{BN}$ gel gradient proved to be a powerful method for the separation of thylakoid megaand supercomplexes in their native state as evidenced by the characterisation of large PSI complexes in control BS and LM treated $M$ maize thylakoids. For quantification of complexes, appropriate sample application, adequate baseline correction, and deconvolution of complexes running in the same band are necessary. To compare the amounts of thylakoid complexes correctly in varying samples, ways of comparison the absolute/relative amounts of complexes and distribution among their different forms are proposed.

\section{Methods}

\section{Plant materials}

Plants used for experiments were as follows. Wheat (Triticum aestivum $\mathrm{L}$. cv. Mv Béres) and maize (Zea mays L. cv. Mv350) were grown in hydroponics ( $1 / 4$ strength Hoagland solution changed every 2 days) for 11 and 14 days, respectively, in a climate chamber (14/10 light/ dark period, photosynthetic photon flux density (PPFD) of 200-250 $\mu \mathrm{mol}$ photons $\mathrm{m}^{-2} \mathrm{~s}^{-1}, 23^{\circ} \mathrm{C}$ and $\sim 75 \%$ relative humidity). Oilseed rape (Brassica napus L. cv. DK Exquisite) was grown under similar conditions in a modified half-strength Hoagland solution [82]. Poplar plants (Populus jacquemontiana Kimura var. glauca Haines cv. Kopeczkii) were grown hydroponically as it is given in Solti et al. [83]. For LM treatment, maize (Zea mays L. cv. Mv NK $333 \mathrm{TC}$ ) seedlings were grown in darkness for 5 days and then greened for $72 \mathrm{~h}$ with continuous illumination of $100 \mu \mathrm{mol}$ photons $\mathrm{m}^{-2} \mathrm{~s}^{-1}$ PPFD. LM (Sigma, $100 \mu \mathrm{g} \mathrm{mL}^{-1}$ ) was added into the nutrient solution $16 \mathrm{~h}$ before the start of illumination and was present during the whole greening process. Other plants were cultivated in the proper soil cultures: Haberlea rhodopensis Friv., shade ecotype at $25 \mu \mathrm{mol}$ photons $\mathrm{m}^{-2} \mathrm{~s}^{-1}$, Primula auricula L. at ambient illumination in greenhouse, and Jatropha curcas L. in the same climate chamber and conditions as mentioned above.

\section{Isolation of thylakoids}

Poplar and H. rhodopensis thylakoids were isolated as it is written in Sárvári and Nyitrai [7] and in Georgieva et al. [84], respectively. Leaves of other plants were homogenised in $50 \mathrm{mM}$ 4-(2-hydroxyethyl)-1-piperazineethanesulphonic acid (HEPES)-KOH, pH 7.0, $330 \mathrm{mM}$ sorbitol, $2 \mathrm{mM}$ ethylenediaminetetraacetic acid (EDTA), $2 \mathrm{mM}$ $\mathrm{MgCl}_{2}, 0.1 \%(\mathrm{w} / \mathrm{V})$ bovine serum albumin (BSA), $0.1 \%(\mathrm{w} / \mathrm{V}) \mathrm{Na}$-ascorbate at $4{ }^{\circ} \mathrm{C}$ for $2 \times 3 \mathrm{~s}$ by Waring blender. The homogenate was filtered through four layers of gauze and two layers of Miracloth ${ }^{\mathrm{TM}}$ (CalbiochemNovabiochem, San Diego, CA, USA). Chloroplasts were immediately pelleted by centrifugation $(1500 \times \mathrm{g}, 5 \mathrm{~min}$, $4{ }^{\circ} \mathrm{C}$ ). The chloroplast pellet was resuspended in washing buffer (50 mM HEPES-KOH pH 7.0, $330 \mathrm{mM}$ sorbitol, $2 \mathrm{mM} \mathrm{MgCl}_{2}$ ). Osmotic shock and removal of $\mathrm{CF}_{1}$ according to Fuad et al. [85] was carried out as described previously [7]. For isolation of BS thylakoids from maize leaves, the material remaining on the filter after a short 


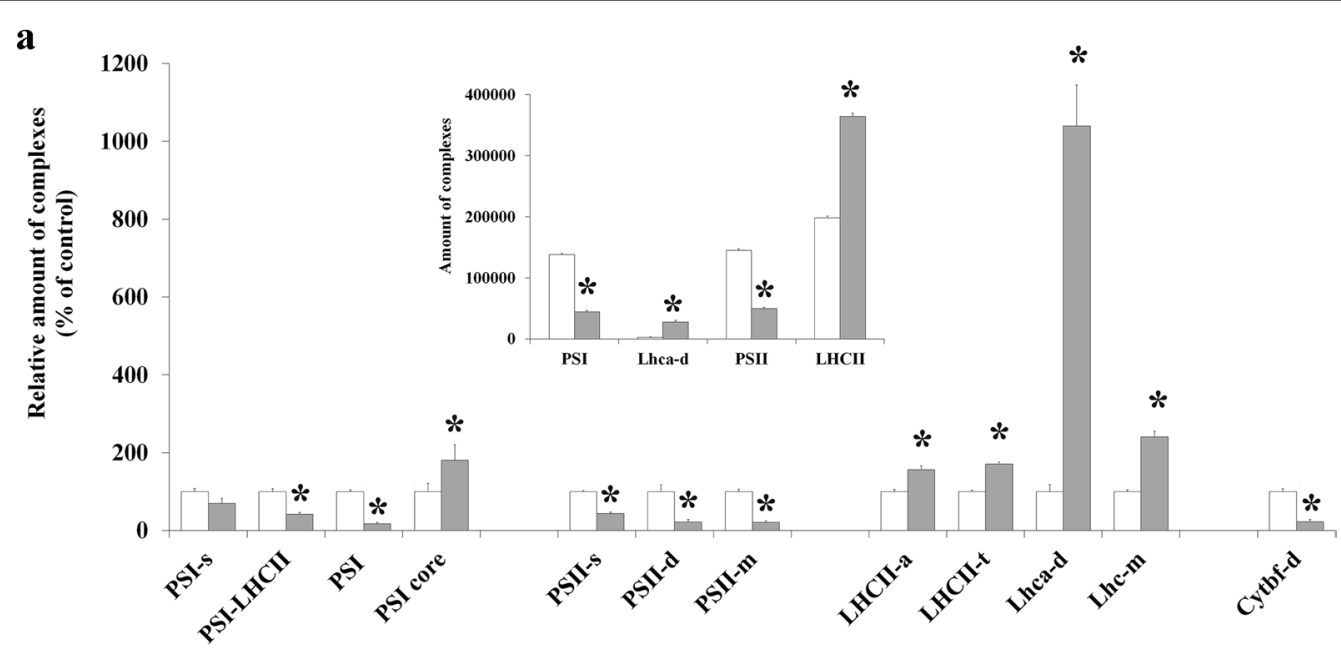

b

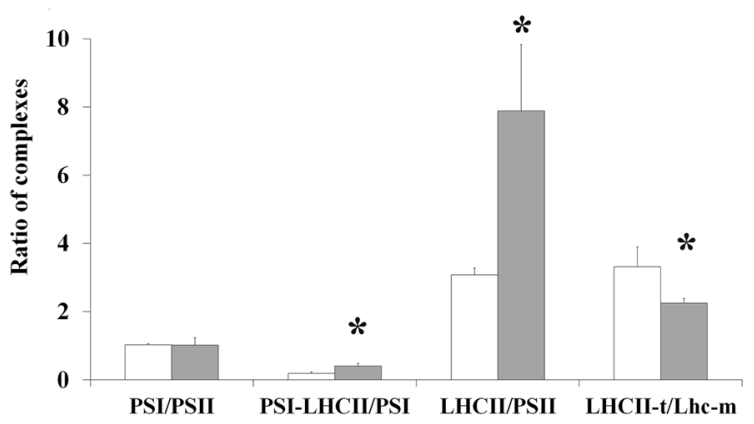

c

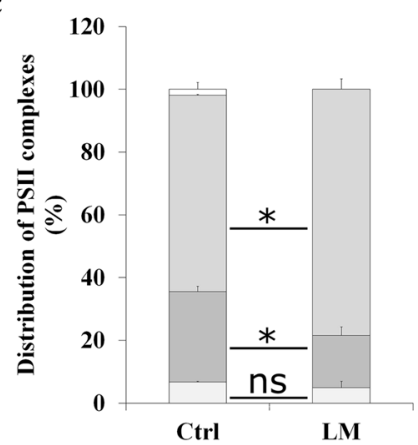

Fig. 9 Amounts, ratios, and distribution of complexes in untreated and lincomycin treated maize mesophyll thylakoids. Complexes are marked as in Fig. 1a, s: supercomplex, d: dimer, m: monomer. Thylakoids were solubilised (500 $\mu \mathrm{g} \mathrm{Chl} \mathrm{mL}{ }^{-1}$ ) using 1\% (w/V) $\beta$-DM plus $1 \%$ (w/V) digitonin and separated in $4.3-12 \%$ BN gel gradient followed by SDS PAGE. a Relative amount of complexes in thylakoids isolated from untreated (white) and lincomycin treated leaves (grey) given as the percentage of controls. Differences were compared using two-way ANOVA with Bonferroni's multiple comparison test $\left[P<0.05\right.$; significance is indicated by stars $\left.\left(^{*}\right)\right]$. Inset: Distribution of total band volumes among the complexes. Differences were compared using multiple t-tests $[P<0.05$; significance is indicated by stars $(*)]$. b Ratio of complexes. The PSI/PSII, PSI-LHCI/PSI, and Lhc-m/LHCII-t ratios were calculated from the BN bands, i.e. PSI and PSII contained the bound antennae, while LHCII/PSII was calculated from the SDS gel pattern: all Lhcb spots and PsbB spots were taken into account (see Additional file 2). Differences were compared using multiple t-tests $[P<0.05$; significance is indicated by stars $\left(^{*}\right)$ ]. c Distribution of PSII complexes (PsbB in SDS PAGE pattern to eliminate the effects of different antenna sizes): PSII-d (light grey), PSII-m (dark grey), PSII-s (medium grey). In controls some CP43-less PSII was also present (white on the top). Differences were compared using multiple t-tests $\left[P<0.05\right.$; significance is indicated by stars $\left.\left({ }^{*}\right)\right]$. In all cases, $n=2 \times 3$ (biological $\times$ technical)

homogenisation to isolate $\mathrm{M}$ plastids was homogenised two times in fresh medium for 30-30 s, then filtered and rinsed, and at last the BS strands remaining on the filter were homogenised further in a mortar, then thylakoid isolation followed as it was mentioned above. Thylakoid samples were stored in $2 \mathrm{mM}$ tris(hydroxymethyl)aminomethane (Tris)-maleate $\mathrm{pH} 7.0,35 \%(\mathrm{w} / \mathrm{V})$ glycerol in liquid nitrogen. They can be stored for years without change under these conditions, but they must be stored in aliquots used once for solubilisation and native PAGE.

\section{Determination of chlorophyll content}

Pigments were extracted from leaf disks or thylakoids with buffered (5 $\mathrm{mM} \mathrm{N}$-(tri(hydroxymethyl)methyl) glycine (Tricine)- $\mathrm{KOH} \mathrm{pH} \mathrm{7.8)} 80 \%(\mathrm{~V} / \mathrm{V})$ acetone at low light. The extracts were centrifuged at $4{ }^{\circ} \mathrm{C}$ with $10,000 \times g$ for $5 \mathrm{~min}$ and measured spectrophotometrically by a UV-VIS spectrophotometer (UV-1601, Shimadzu, Japan). Chl content was calculated according to Porra et al. [86].

PAGE methods (see also Additional file 2: BN-solubilisation and evaluation; Additional file 5: Gel preparations and buffers).

To separate thylakoid complexes, gel electrophoresis was performed under native conditions by BN-PAGE [12] based on the method of Schagger and von Jagow [11] but using Mini-Protean apparatus (BioRad) and 4\% $(\mathrm{w} / \mathrm{V})$ stacking and $5-12 \%$ or $4.3-12 \%$ or $4.3-8 \%(\mathrm{w} / \mathrm{V})$ 
acrylamide gradient separating gels of $1.5 \mathrm{~mm}$, all containing $8.7 \%(\mathrm{w} / \mathrm{V})$ glycerol. After washing by $10 \mathrm{~min}$ centrifugation with $10,000 \times g$ in washing buffer $(50 \mathrm{mM}$ BisTris- $\mathrm{HCl} \mathrm{pH} 7.0,330 \mathrm{mM}$ sorbitol, $250 \mu \mathrm{g} \mathrm{mL}$

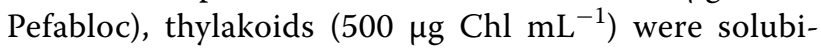
lised in solubilising buffer $(750 \mathrm{mM}$ aminocaproic acid [ACA], $50 \mathrm{mM}$ Bis-Tris, $\mathrm{pH}$ 7.0, $0.5 \mathrm{mM}$ EDTA) with $1 \%(\mathrm{w} / \mathrm{V}) \beta$-DM (SIGMA) plus $1 \%(\mathrm{w} / \mathrm{V})$ digitonin (SERVA) or other detergents/mixtures including DS (Calbiochem). Two-fold concentrated stock $(2 \times \mathrm{SB})$ supplemented by a corresponding volume of deionised water to the volume of the thylakoid samples was applied in order to achieve the final concentration of the solubilisation buffer and Chls. After resuspending the thylakoid pellet with pipette, solubilisation was performed after adding the chosen detergent (see also Additional file 2, sheet "solubilisation") on ice for $30 \mathrm{~min}$ with three short vortexing meantime. After 15 min centrifugation with $18,000 \times g$ at $4{ }^{\circ} \mathrm{C}$, the supernatant was supplemented with $1 / 5$ volume of glycerol for CN PAGE or 5\% (w/V) Serva Blue G (SBG) dissolved in $500 \mathrm{mM}$ ACA for BN PAGE, and 10-20 $\mu \mathrm{L}$ of the solubilised material was applied per lanes. To remove residual ammonium persulphate from the wells, sample places were rinsed with cathode buffer for CN PAGE and cathode buffer containing 5-times diluted SBG (that enables a more convenient sample application) for BN PAGE before sample loading. CN PAGE was performed as BN PAGE except that cathode buffer contained $0.02 \%(\mathrm{w} / \mathrm{V}) \beta-\mathrm{DM}$ and $0.05 \%(\mathrm{w} / \mathrm{V})$ sodium deoxycholate (DOC) instead of SBG.

For the second-dimensional BN PAGE mainly according to Rantala et al. [17], gel strips were solubilised using $1 \%(\mathrm{w} / \mathrm{V}) \beta$-DM in solubilising buffer for $30 \mathrm{~min}$ in ice by shaking, then rinsed and placed to the top of a gradient gel $(4.3-12 \%)$ in solubilising buffer containing $1 \%(\mathrm{w} / \mathrm{V}) \beta$-DM, $5 \%(\mathrm{w} / \mathrm{V}) \mathrm{SBG}$, and $0.5 \%(\mathrm{w} / \mathrm{V})$ agarose. Agarose was dissolved by heating. Warm agarose cooled down immediately during pipetting around the gel slice. Cathode buffer contained $0.02 \%(w / V)$ SBG plus $0.02 \%(\mathrm{w} / \mathrm{V}) \beta$-DM.

Electrophoresis was carried out at $6{ }^{\circ} \mathrm{C}$ with a maximum of $5 \mathrm{~mA}$ per gel $\left(1 \mathrm{~cm}^{2}\right.$ gel surface $)$ with constant voltage of $50 \mathrm{~V}(30 \mathrm{~min}), 100 \mathrm{~V}(30 \mathrm{~min}), 150 \mathrm{~V}$ (30 $\mathrm{min}$ ), and after changing the upper buffer to one without SBG or blue cathode buffer with $1000 \times$ diluted SBG (it was more convenient) in BN PAGE and using the original cathode buffer in CN PAGE, with $200 \mathrm{~V}$ (about $2 \mathrm{~h}$ ).

$\mathrm{NAD}(\mathrm{P}) \mathrm{H}$ dehydrogenase in gel activity was detected as in Quiles et al. [87].

The polypeptide pattern of thylakoids and thylakoid complexes was determined by SDS-PAGE according to Laemmli [52] but modified by using $5 \%$ stacking and 10-18\% linear gradient separating gels, all containing $8.7 \%(\mathrm{w} / \mathrm{V})$ glycerol. To obtain the polypeptide patterns of thylakoid complexes, gel strips of about $3 \mathrm{~mm}$ wide were cut out of BN-PAGE lanes and attached to the top of the denaturing gel in solubilising buffer containing $0.5 \%(\mathrm{w} / \mathrm{V})$ agarose. Proteins were separated in the above-mentioned apparatus with a constant current of $20 \mathrm{~mA}$ per gel for about $2 \mathrm{~h}$. Following the electrophoresis, gels were stained with the Blue-Silver method [88]. Some polypeptide spots determining the identity of the complexes unequivocally could be recognised on the basis of previous MS determinations of bands run under similar conditions as it was done in Basa et al. [28].

The gels were scanned using an Epson Perfection V750 PRO scanner. Densitometry analysis of gels (i.e. integration of the density of pixels as band volumes on grayscale, not adjusted images) was carried out using the Phoretix image analysis software (Phoretix International, Newcastle-upon-Tyne, UK).

\section{Determination of protein content}

Protein standards (bovine serum albumin, $66 \mathrm{kDa}$; ovalbumin, $45 \mathrm{kDa}$; glyceraldehyde-3-phosphate dehydrogenase, $36 \mathrm{kDa}$; carbonic anhydrase, $29 \mathrm{kDa}$; trypsinogen, $24 \mathrm{kDa}$; trypsin inhibitor, $20 \mathrm{kDa}$; $\alpha$-lactalbumin, $14 \mathrm{kDa}$ ) containing $5 \mu \mathrm{g}$ protein were run together with solubilised (30 min at room temperature) thylakoids in SDS gels, and their total lane volumes were compared.

\section{Western blot}

Membrane proteins separated by SDS-PAGE were transferred to HyboundTM-C Extra (Amersham-Pharmacia, Piscataway, NY, USA) nitrocellulose membranes in $25 \mathrm{mM}$ Tris $\mathrm{pH}$ 8.3, $192 \mathrm{mM}$ glycine, 20\% (V/V) methanol and $0.02 \%(\mathrm{w} / \mathrm{V})$ SDS at $4{ }^{\circ} \mathrm{C}$ using $90 \mathrm{~V}$ constant voltage $(<0.4 \mathrm{~A})$ for $3 \mathrm{~h}$. Membranes were decorated with rabbit polyclonal antibodies against Lhcb-s (a gift from Dr. Udo Johanningmeier, Bohum Universität, Germany) or with Agrisera AG (Vännäs, Sweden) antibodies against Lhca1 (AS01 005), Lhca2 (AS01 006), Lhca3 (AS01 007) and Lhca4 (AS01 008]). Antibody detection was carried out as in Solti et al. [89].

\section{Proteomics \\ Sample preparation}

Gel band or spots cut out from BN or SDS gels were destained in $25 \mathrm{mM}$ ammonium bicarbonate (AMBIC) $/ 50 \%$ acetonitrile $(\mathrm{ACN})$, reduction was performed in $10 \mathrm{mM}$ tris-(2-carboxyethyl)-phosphine at $56{ }^{\circ} \mathrm{C}$ for $30 \mathrm{~min}$, then alkylation with $55 \mathrm{mM}$ iodoacetamide at room temperature for $30 \mathrm{~min}$ in dark. Gel pieces were washed using $25 \mathrm{mM}$ AMBIC/50\% $\mathrm{ACN}$ and dried 
using SpeedVac. Gel pieces were then rehydrated using $5 \mathrm{ng} \mathrm{LL}^{-1}$ trypsin in $25 \mathrm{mM}$ AMBIC. Digestion was performed overnight at $37^{\circ} \mathrm{C}$. Reaction was stopped by adding $2 \%$ formic acid (FA) to the digestion solution. Gel pieces were washed and sonicated in $50 \% \mathrm{ACN} / 1 \% \mathrm{FA}$. Solution containing the resulting peptides were dried in SpeedVac, then redissolved and cleaned on C18 Spin Columns. Eluted samples were dried in SpeedVac and kept at $-20{ }^{\circ} \mathrm{C}$. Samples were reconstituted in $25 \mu \mathrm{L}$ $2 \% \mathrm{ACN}, 0.1 \%$ FA before injection to a Waters Acquity I-Class UPLC system connected to Waters Select Series Cyclic IMS (Waters Corporation, Milford, UK). For peptide separation, samples were loaded on Waters Acquity Premier CSH column $(150 \times 1 \mathrm{~mm}, 1.7 \mu \mathrm{m})$ for multistep gradient elution. Mobile phase (A) was composed of $0.1 \%$ FA in water, mobile phase (B) was composed of $0.1 \% \mathrm{FA}$ in $\mathrm{ACN}$. The elution method at flow rate $20 \mu \mathrm{L} \mathrm{min}{ }^{-1}$ included the following: $1 \mathrm{~min}: 5 \% \mathrm{~B}, 45 \mathrm{~min}$ : $35 \%$ B, 46 min: $85 \%$ B at $45{ }^{\circ} \mathrm{C}$. MS data acquisition was performed with the following parameters: $\mathrm{m} / \mathrm{z} 50-2000$, V-mode, scan time: $0.5 \mathrm{~s}$, single Lock Mass: leucine enkephalin. $M S^{\mathrm{E}}$ fragmentation was performed in the trap: low energy: $6 \mathrm{~V}$, high energy: ramping $19-45 \mathrm{~V}$.

\section{Data analysis}

Data were analysed qualitatively using Waters ProteinLynx Global Server software. Parent and fragment ion tolerance was set to $20 \mathrm{ppm}$ and $30 \mathrm{ppm}$ respectively. Digestion enzyme was trypsin, missed cleavages were set to 2. Carbamidomethyl modification on cysteines was set as fixed modification, for variable modifications methionine oxidation was allowed. False discovery rate was $2 \%$, and only proteins with minimal probability of $95 \%$ were counted. First, data were run in SwissProt database in order to collect contaminants. Then, analysis was performed in Maize and PSI-NDH databases (acquired form UniProtKB 03. 2021.), supplemented with the contaminant protein list. Processing parameters were the following: low energy threshold: 200 counts; elevated energy threshold: 20 counts; minimum fragment ion matches per peptide: 3 ; minimum fragment ion matches per protein: 7; minimum peptide matches per protein: 2 .

\section{Statistics}

Statistical analysis was performed using Prism v. 8.0.1 (GraphPad Software, Inc., San Diego, CA, United States). Methodology details are given in each figure legend. In general, data obtained from 2-3 separate isolations with (2)-3 technical replicates were compared except methodology data where only technical repetitions (3-6) were used to exclude differences among samples. Percentage data in Fig. 9a were compared using two-way ANOVA with Bonferroni's multiple comparison test $(P<0.05)$ and in Additional file 1: Fig. S10 using one-way ANOVA with Tukey's multiple comparison test $(P<0.05)$. Groups containing two members (Figs. 5g, 9a-inset, b, c) were compared with multiple $\mathrm{t}$-tests $(P<0.05)$, and those with $>2$ members (Figs. 3b, 7b, Additional file 1: Figs. S6 and S7) were compared using one-way ANOVA with Tukey's multiple comparison test $(P<0.05)$. Comparisons were made separately in the different groups.

\begin{abstract}
Abbreviations
ACA: Aminocaproic acid; ACN: Acetonitrile; AMBIC: Ammonium bicarbonate; ATPS: ATP synthase; BN: Blue Native; BS: Bundle sheath; $\mathrm{CF}_{1}$ : Coupling factor 1 Chl: Chlorophyll; CN: Clear Native; CP: Chlorophyll-protein; Cyt: Cytochrome; d: Dimer; $\alpha / \beta$-DM: $n$-Dodecyl- $/$ / $\beta$-D-maltoside; DOC: Sodium deoxychlolate; DS: n-Dodecyl-sucrose; EDTA: Ethylenediaminetetraacetic acid; FA: Formic acid; HEPES: 4-(2-Hydroxyethyl)-1-piperazineethanesulphonic acid; Lhc/LHC: Light-harvesting complex; LHCII-a: LHCII assembly complex containing CP29, CP24 and M-LHCII trimer; LM: Lincomycin; M: Mesophyll; m: Monomer; mc: Megacomplex; MS: Mass spectrometry; NDH: NADH dehydrogenase-like complex; PAGE: Polyacrylamide gel electrophoresis; PS: Photosystem; s: Supercomplex; SBG: Serva Blue G; SDS: Sodium dodecyl sulphate; t: Trimer; Tricine: $\mathrm{N}$-(Tri(hydroxymethyl)methyl)glycine; Tris: Tris(hydroxymethyl)aminomethane.
\end{abstract}

\section{Supplementary Information}

The online version contains supplementary material available at https://doi. org/10.1186/s13007-022-00858-2.

Additional file 1. Additional figures S1-S12.

Additional file 2. BN-solubilisation and evaluation

Additional file 3 . MS results 1 .

Additional file 4. MS results 2 .

Additional file 5. Gel preparations and buffers.

\section{Acknowledgements}

We are grateful to Györgyi Balogh (Department of Plant Physiology and Molecular Plant Biology, ELTE Eötvös Loránd University) for the skilful assistance in gel electrophoresis and Csilla Gergely (Department of Plant Anatomy, ELTE Eötvös Loránd University) for help with the electron microscopic sample preparation, and for the antibody gifts from Udo Johanningmeier (LHCII) and Gergana Mihailova (Lhca-s). Katya Georgieva and Gergana Mihailova (Institute of Plant Physiology and Genetics, BAS, Sofia, Bulgaria) are acknowledged for providing plant material from Haberlea rhodopensis. We thank Tibor Janda (Centre for Agricultural Research, Agricultural Institute, ELKH, Martonvásár, Hungary) for maize seeds. Jatropha curcas seeds were obtained as generous gifts from Helena Sapeta and Margarida Oliveira (ITQB, NOVA, Portugal). We are also very grateful to our reviewers whose helpful advices greatly contributed to the perfection of the manuscript.

\section{Authors' contributions}

ÉS conceived and designed the study, performed the experiments and evaluated the results. KS performed TEM analysis and prepared and provided some experimental plant material. GS and GG performed mass spectrometry analysis. MS-K performed the statistical analysis. ÉS and ÁS wrote the paper. All authors read and approved the final manuscript.

\section{Funding}

Open access funding provided by Eötvös Loránd University. The work was supported by the grants of the National Research, Development and Innovation Office (OTKA FK124748 to K.S.) and the Lendület (Momentum) Program and Ion Mobility Mass Spectrometry Research Group of the Hungarian Academy of 
Sciences (to G.S.). K.S. was supported by the János Bolyai Research Scholarship of the Hungarian Academy of Sciences. M.S-K. was supported by the New National Excellence Program of the Ministry of Technology and Innovation Hungary (ÚNKP-20-3-I-ELTE-862). Purchase of the mass spectrometer was funded by the Synthesis + ELTE Thematic Excellence Programme supported by the Hungarian Ministry for Innovation and Technology. Lhca antibodies were purchased in the frame of Bulgarian National Science Fund, Ministry of Education and Science (Project KП-06-H21/8 to K. Georgieva) in which we used the method to follow the recovery of cold-stressed Haberlea rhodopensis. Investigations were performed in the frame of the Joint Research Program of the Bulgarian and Hungarian Academies of Sciences (NKM 2018-107).

\section{Availability of data and materials}

All data generated or analysed during this study are included in this published article.

\section{Declarations}

\section{Ethics approval and consent to participate}

Not applicable.

\section{Consent for publication}

All authors agreed to publish this manuscript.

\section{Competing interests}

The authors declare that they have no competing interests.

\section{Author details}

'Department of Plant Physiology and Molecular Plant Biology, Institute of Biology, Faculty of Science, ELTE Eötvös Loránd University, Pázmány Péter sétány 1/C, Budapest 1117, Hungary. ${ }^{2}$ MTA-ELTE Lendület lon Mobility Mass Spectrometry Research Group, Institute of Chemistry, Faculty of Science, ELTE Eötvös Loránd University, Pázmány Péter sétány 1/A, Budapest 1117, Hungary. ${ }^{3}$ Doctoral School of Biology, Institute of Biology, Faculty of Science, ELTE Eötvös Loránd University, Pázmány Péter sétány 1/C, Budapest 1117, Hungary. ${ }^{4}$ Department of Plant Anatomy, Institute of Biology, Faculty of Science, ELTE Eötvös Loránd University, Pázmány Péter sétány 1/C, Budapest 1117, Hungary.

\section{Received: 20 October 2021 Accepted: 12 February 2022}

Published online: 03 March 2022

\section{References}

1. Rexroth S, Meyer zu Tittingdorf JMW, Krause F, Dencher NA, Seelert H. Thylakoid membrane at altered metabolic state: challenging the forgotten realms of the proteome. Electrophoresis. 2003;24:2814-23.

2. Tomizioli M, Lazar C, Brugière S, Burger T, Salvi D, Gatto L, et al. Deciphering thylakoid sub-compartments using a mass spectrometry-based approach. Mol Cell Proteomics. 2014;13:2147-67.

3. Reisinger $V$, Eichacker LA. How to analyze protein complexes by $2 \mathrm{D}$ blue native SDS-PAGE. Proteomics. 2007;7:6-16.

4. Anderson JM, Waldron JC, Thorne SW. Chlorophyll-protein complexes of spinach and barley thylakoids: spectral characterization of six complexes resolved by an improved electrophoretic procedure. FEBS Lett. 1978;92:227-33

5. Peter GF, Thornber JP. Biochemical composition and organization of higher plant photosystem II light-harvesting pigment-proteins. J Biol Chem. 1991;266:16745-54.

6. Allen KD, Staehelin LA. Resolution of 16 to 20 chlorophyll-protein complexes using a low ionic strength native green gel system. Anal Biochem. 1991;194:214-22.

7. Sárvári É, Nyitrai P. (1994), Separation of chlorophyll-protein complexes by deriphat polyacrylamide gradient gel electrophoresis. Electrophoresis. 1994;15:1068-71.

8. de Bianchi S, Dall'Osto L, Tognon G, Morosinotto T, Bassi R. Minor antenna proteins CP24 and CP26 affect the interactions between photosystem II subunits and the electron transport rate in grana membranes of Arabidopsis. Plant Cell. 2008;20:1012-28.
9. Caffarri S, Kouřil R, Kereïche S, Boekema EJ, Croce R. Functional architecture of higher plant photosystem II supercomplexes. EMBO J. 2009;28:3052-63.

10. Galka P, Santabarbara S, Khuong TTH, Degand H, Morsomme P, Jennings $\mathrm{RC}$, et al. Functional analyses of the plant photosystem I-light-harvesting complex II supercomplex reveal that light-harvesting complex II loosely bound to photosystem II is a very efficient antenna for photosystem I in state II. Plant Cell. 2012;24:2963-78.

11. Schägger H, von Jagow G. Blue native electrophoresis for isolation of membrane protein complexes in enzymatically active form. Anal Biochem. 1991;199:223-31.

12 Kügler M, Jänsch L, Kruft V, Schmitz UK, Braun HP. Analysis of the chloroplast protein complexes by blue-native polyacrylamide gel electrophoresis (BN-PAGE). Photosynth Res. 1997;53:35-44.

13. Eubel $H$, Braun HP, Millar AH. Blue-native PAGE in plants: a tool in analysis of protein-protein interactions. Plant Methods. 2005;16:11.

14. Wittig I, Braun HP, Schägger H. Blue native PAGE. Nat Protoc. 2006;1:418-28.

15. Krause F. Detection and analysis of protein-protein interactions in organellar and prokaryotic proteomes by native gel electrophoresis: (Membrane) protein complexes and supercomplexes. Electrophoresis. 2006;27:2759-81.

16. Järvi S, Suorsa M, Paakkarinen V, Aro E-M. Optimized native gel systems for separation of thylakoid protein complexes: novel super- and megacomplexes. Biochem J. 2011;439:207-14.

17. Rantala M, Tikkanen M, Aro E-M. Proteomic characterization of hierarchical megacomplex formation in Arabidopsis thylakoid membrane. Plant J. 2017;92:951-62.

18. D'Amici GM, Timperio AM, Zolla L. Coupling of native liquid phase isoelectrofocusing and blue native polyacrylamide gel electrophoresis: a potent tool for native membrane multiprotein complex separation. J Proteome Res. 2008;7:1326-40.

19. Chen YE, Su YQ, Mao HT, Wu N, Zhu F, Yuan M, et al. Terrestrial plants evolve highly assembled photosystem complexes in adaptation to light shifts. Front Plant Sci. 2018;19:1811.

20. Rokka A, Suorsa M, Saleem A, Battchikova N, Aro E-M. Synthesis and assembly of thylakoid protein complexes: multiple assembly steps of photosystem II. Biochem J. 2005;388:159-68.

21. Wittenberg G, Järvi S, Hojka M, Tóth SZ, Meyer EH, Aro E-M, et al. Identification and characterization of a stable intermediate in photosystem I assembly in tobacco. Plant J. 2017;90:478-90.

22. Suorsa M, Rantala M, Mamedov F, Lespinasse M, Trotta A, Grieco M, et al. Light acclimation involves dynamic re-organization of the pigmentprotein megacomplexes in non-appressed thylakoid domains. Plant J. 2015:84:360-73.

23. Ferroni L, Suorsa M, Aro E-M, Baldisserotto C, Pancaldi S. Light acclimation in the lycophyte Selaginella martensii depends on changes in the amount of photosystems and on the flexibility of the light-harvesting complex II antenna association with both photosystems. New Phytol. 2016;211:554-68

24. Bielczynski LW, Schansker G, Croce R. Effect of light acclimation on the organization of photosystem II super- and sub-complexes in Arabidopsis thaliana. Front Plant Sci. 2016;7:105

25. Rogowski P, Wasilewska-Dębowska W, Krupnik T, Drożak A, Zienkiewicz $M$, Krysiak M, et al. Photosynthesis and organization of maize mesophyll and bundle sheath thylakoids of plants grown in various light intensities. Environ Exp Bot. 2019;162:72-86.

26. Andaluz S, López-Millán AF, De las Rivas J, Aro E-M, Abadía J, Abadía A. Proteomic profiles of thylakoid membranes and changes in response to iron deficiency. Photosynth Res. 2006;89:141-55.

27. Fagioni M, D'Amici GM, Timperio AM, Zolla L. Proteomic analysis of multiprotein complexes in the thylakoid membrane upon cadmium treatment. J Proteome Res. 2009;8:310-26.

28. Basa B, Lattanzio G, Solti Á, Tóth B, Abadía J, Fodor F, et al. Changes induced by cadmium stress and iron deficiency in the composition and organization of thylakoid complexes in sugar beet (Beta vulgaris L.). Environ Exp Bot. 2014;101:1-11.

29. Sárvári É, Mihailova G, Solti Á, Keresztes Á, Velitchkova M, Georgieva K. Comparison of thylakoid structure and organization in sun and shade Haberlea rhodopensis populations under desiccation and rehydration. J Plant Physiol. 2014;171:1591-600. 
30. Solti Á, Sárvári É, Tóth B, Mészáros I, Fodor F. Incorporation of iron into chloroplasts triggers the restoration of cadmium induced inhibition of photosynthesis. J Plant Physiol. 2016;202:97-106.

31. Mihailova G, Solti Á, Sárvári É, Keresztes Á, Rapparini F, Velitchkova M, et al. Freezing tolerance of photosynthetic apparatus in the homoiochlorophyllous resurrection plant Haberlea rhodopensis. Environ Exp Bot. 2020;178:104157.

32. Chen X, Zhang W, Xie Y, Lu W, Zhang R. Comparative proteomics of thylakoid membrane from a chlorophyll $b$-less rice mutant and its wild type. Plant Sci. 2007;173:397-407.

33. Kim EH, Li XP, Razeghifard R, Anderson JM, Niyogi KK, Pogson BJ, et al. The multiple roles of light-harvesting chlorophyll $a / b$-protein complexes define structure and optimize function of Arabidopsis chloroplasts: a study using two chlorophyll $b$-less mutants. Biochim Biophys Acta. 2009; 1787:973-84.

34. Takabayashi A, Kurihara K, Kuwano M, Kasahara Y, Tanaka R, Tanaka A. The oligomeric states of the photosystems and the light-harvesting complexes in the Chl b-less mutant. Plant Cell Physiol. 2011;52:2103-14.

35. Benson $S$, Maheswaran $P$, Ware $M$, Hunter $C N$, Horton $P$, Jansson $S$, et al. An intact light harvesting complex I antenna system is required for complete state transitions in Arabidopsis. Nat Plants. 2015;1:15176.

36. Grieco M, Suorsa M, Jajoo A, Tikkanen M, Aro E-M. Light-harvesting II antenna trimers connect energetically the entire photosynthetic machinery_including both photosystems II and I. Biochim Biophys Acta. 2015;1847:607-19.

37. Takabayashi A, Kadoya R, Kuwano M, Kurihara K, Ito H, Tanaka R, et al. Protein co-migration database (PCOM -DB) for Arabidopsis thylakoids and Synechocystis cells. Springerplus. 2013;2:148.

38. Heinemeyer J, Eubel $H$, Wehmhöner $D$, Jänsch $L$, Braun H-P. Proteomic approach to characterize the supramolecular organization of photosystems in higher plants. Phytochemistry. 2004;65:1683-92.

39. Pan X, Ma J, Su X, Cao P, Chang W, Liu Z, et al. Structure of the maize photosystem I supercomplex with light-harvesting complexes I and II. Science. 2018;360:1109-13.

40. Kourili R, Zygadlo A, Arteni AA, de Wit CD, Dekker JP, Jensen PE, et al. Structural characterization of a complex of photosystem I and light-harvesting complex II of Arabidopsis thaliana. Biochemistry. 2005:44:10935-40.

41. Wientjes $E$, van Amerongen $H$, Croce R. LHCIl is an antenna of both photosystems after long-term acclimation. Biochim Biophys Acta. 2013;1827:420-6.

42. Bell AJ, Frankel LK, Bricker TM. High yield non-detergent isolation of photosystem I-light-harvesting chlorophyll II membranes from spinach thylakoids: Implications for the organization of the PSI antennae in higher plants. J Biol Chem. 2015;290:18429-37.

43. Yadav KNS, Semchonok DA, Nosek L, Kourïl R, Fucile G, Boekema EJ, et al. Supercomplexes of plant photosystem I with cytochrome $b_{6} f$, lightharvesting complex II and NDH. Biochim Biophys Acta. 2017;1858:12-20.

44. Bos I, Bland KM, Tian L, Croce R, Frankel LK, van Amerongen H, et al. Multiple LHCII antennae can transfer energy efficiently to a single Photosystem I. Biochim Biophys Acta. 2017;1858:371-8.

45. Schwarz EM, Tietz S, Froehlich JE. Photosystem I-LHCII megacomplexes respond to high light and aging in plants. Photosynth Res. 2018;136:107-24.

46. Yokono M, Takabayashi A, Kishimoto J, Fujita T, Iwai M, Murakami A, et al. The PSI-PSII megacomplex in green plants. Plant Cell Physiol. 2019;60:1098-108.

47. Iwai M, Takizawa K, Tokutsu R, Okamuro A, Takahashi Y, Minagawa J. Isolation of the elusive supercomplex that drives cyclic electron flow in photosynthesis. Nature. 2010:464:1210-3.

48. Kouřil R, Strouhal O, Nosek L, Lenobel R, Chamrád I, Boekema EJ, et al. Structural characterization of a plant photosystem I and NAD(P)H dehydrogenase supercomplex. Plant J. 2014;77:568-76.

49. Peng L, Shimizu H, Shikanai T. The chloroplast NAD(P)H dehydrogenase complex interacts with photosystem I in Arabidopsis. J Biol Chem. 2008:283:34873-9.

50. Peng L, Fukao Y, Fujiwara M, Takami T, Shikanai T. Efficient operation of $\mathrm{NAD}(\mathrm{P}) \mathrm{H}$ dehydrogenase requires supercomplex formation with photosystem I via minor LHCl in Arabidopsis. Plant Cell. 2009;21:3623-40.
51. Granvogl B, Zoryan M, Plöscher M, Eichacker LA. Localization of 13 onehelix integral membrane proteins in photosystem II subcomplexes. Anal Biochem. 2008;383:279-88.

52. Laemmli UK. Cleavage of structural proteins during assembly of the head of bacteriophage T4. Nature. 1970;227:680-5.

53. Schägger H. Tricine-SDS-PAGE. Nat Protoc. 2006;1:16-22.

54. Koochak H, Puthiyaveetil S, Mullendore DL, Li M, Kirchhoff H. The structural and functional domains of plant thylakoid membranes. Plant $\mathrm{J}$. 2019:97:412-29.

55. Hatch MD. C4 photosynthesis: a unique elend of modified biochemistry, anatomy and ultrastructure. Biochim Biophys Acta. 1987;895:81-106.

56. Josten JJ, Allen PM. The mode of action of lincomycin. Biochem Biophys Res Commun. 1964;14:241-4.

57. Ellis RJ. Inhibition of chloroplast protein synthesis by lincomycin and 2-(4-methyl-2,6-dinitroanilino)-N-methylpropionamide. Phytochemistry. 1975;14:89-93.

58. Mulo P, Pursiheimo S, Hou CX, Tyystjärvi T, Aro E-M. Multiple effects of antibiotics on chloroplast and nuclear gene expression. Funct Plant Biol. 2003;30:1097-103.

59. Gáspár L, Sárvári É, Morales F, Szigeti Z. Presence of 'PSI free' LHCl and monomeric LHCII and subsequent effects on fluorescence characteristics in lincomycin treated maize. Planta. 2006;223:1047-57.

60. Crepin A, Santabarbara S, Caffarri S. Biochemical and spectroscopic characterization of highly stable photosystem II supercomplexes from Arabidopsis. J Biol Chem. 2016;291:19157-71.

61 Pagliano C, Barera S, Chimini F, Saracco G, Barber J. Comparison of the $a$ and $\beta$ isomeric forms of the detergent $n$-dodecyl-D-maltoside for solubilizing photosynthetic complexes from pea thylakoid membranes. Biochim Biophys Acta. 2012;1817:1506-15.

62. Nosek L, Semchonok D, Boekema EJ, llík P, Kouřil R. Structural variability of plant photosystem II megacomplexes in thylakoid membranes. Plant J. 2017:89:104-11.

63. Behrens C, Blume C, Senkler M, Eubel H, Peterhänsel C, Braun H-P. The 'protein complex proteome' of chloroplasts in Arabidopsis thaliana. J Proteomics. 2013:91:73-83.

64 Romanowska E, Kargul J, Powikrowska M, Nield J, Drozak A, Pokorska B. Structural organization of photosynthetic apparatus in agranal chloroplasts of maize metabolism and bioenergetics. J Biol Chem. 2008;283:26037-46.

65. Burrows PA, Sazanov LA, Svab Z, Maliga P, Nixon PJ. Identification of a functional respiratory complex in chloroplasts through analysis of tobacco mutants containing disrupted plastid ndh genes. EMBO J. 1998;17:868-76.

66. Darie CC, Biniossek ML, Winter V, Mutschler B, Haehnel W. Isolation and structural characterization of the Ndh complex from mesophyll and bundle sheath chloroplasts of Zea mays. FEBS J. 2005;272:2705-16.

67. Majeran W, Zybailov B, Ytterberg AJ, Dunsmore J, Sun Q, van Wijk KJ. Consequences of $\mathrm{C}_{4}$ differentiation for chloroplast membrane proteomes in maize mesophyll and bundle sheath cells. Mol Cell Proteomics. 2008;7:1609-38.

68. Takabayashi A, Kishine M, Asada K, Endo T, Sato F. Differential use of two cyclic electron flows around photosystem I for driving $\mathrm{CO}_{2}$-concentration mechanism in $\mathrm{C}_{4}$ photosynthesis. Proc Natl Acad Sci USA. 2005;102:16898-903.

69. Darie CC, De Pascalis L, Mutschler B, Haehnel W. Studies of the Ndh complex and photosystem II from mesophyll and bundle sheath chloroplasts of the $\mathrm{C}_{4}$-type plant Zea mays. J Plant Physiol. 2006;163:800-8.

70. Otani T, Kato Y, Shikanai T. Specific substitutions of light-harvesting complex I proteins associated with photosystem I are required for supercomplex formation with chloroplast NADH dehydrogenase-like complex. Plant J. 2018;94:122-30

71. Shen L, Tang K, Wang W, Wang C, Wu H, An MS, et al. Architecture of the chloroplast PSI-NDH supercomplex in Hordeum vulgare. Nature. 2021. https://doi.org/10.1038/s41586-021-04277-6.

72. Shikanai T. Chloroplast NDH: a different enzyme with a structure similar to that of respiratory NADH dehydrogenase. Biochim Biophys Acta. 2016;1857:1015-22.

73. Peltier G, Aro E-M, Shikanai T. NDH-1 and NDH-2 plastoquinone reductases in oxygenic photosynthesis. Annu Rev Plant Biol. 2016;67:55-80. 
74. Pan X, Cao D, Xie F, Xu F, Su X, Mi H, et al. Structural basis for electron transport mechanism of complex I-like photosynthetic NAD(P)H dehydrogenase. Nat Commun. 2020;11:610.

75. Yamamoto H, Peng L, Fukao Y, Shikanai T. An Src homology 3 domainlike fold protein forms a ferredoxin binding site for the chloroplast $\mathrm{NADH}$ dehydrogenase-like complex in Arabidopsis. Plant Cell. 2011:23:1480-93

76. Klodmann J, Senkler M, Rode C, Braun H-P. Defining the protein complex proteome of plant mitochondria. Plant Physiol. 2011;157:587-98.

77. Klusch N, Senkler J, Yildiz Ö, Kühlbrandt W, Braun H-P. A ferredoxin bridge connects the two arms of plant mitochondrial complex I. Plant Cell. 2021;33:2072-91.

78. Xu M, Shi N, Li Q, Mi H. An active supercomplex of NADPH dehydrogenase mediated cyclic electron flow around Photosystem I from the panicle chloroplast of Oryza sativa. Acta Biochim Biophys Sinica. 2014;46:757-65.

79. Crepin A, Kučerová Z, Kosta A, Durand E, Caffarri S. Isolation and characterization of a large photosystem I-light-harvesting complex II supercomplex with an additional Lhca1_a4 dimer in Arabidopsis. Plant J. 2020;102:398-409.

80. Sárvári É, Halász G, Török S, Láng F. Light-induced fluorescence decay during the greening of normal and lincomycin-treated maize leaves. Planta. 1978;141:135-9.

81. Tóth TN, Rai N, Solymosi K, Zsiros O, Schröder WP, Garab G, et al. Fingerprinting the macro-organisation of pigment-protein complexes in plant thylakoid membranes in vivo by circular-dichroism spectroscopy. Biochim Biophys Acta. 2016;1857:1479-89.

82. Müller B, Kovács K, Pham HD, Kavak Y, Pechoušek J, Machala L. Chloroplasts preferentially take up ferric-citrate over iron-nicotianamine complexes in Brassica napus. Planta. 2019;249:751-63.

83. Solti Á, Gáspár L, Mészáros I, Szigeti Z, Lévai L, Sárvári É. Impact of iron supply on the kinetics of recovery of photosynthesis in Cd-stressed poplar (Populus glauca). Ann Bot. 2008;102:771-82.

84. Georgieva K, Röding A, Büchel C. Changes in some thylakoid membrane proteins and pigments upon desiccation of the resurrection plant Haberlea rhodopensis. J Plant Physiol. 2009;166:1520-8.

85. Fuad N, Day DA, Ryrie IJ, Thorne SW. A photosystem II light-harvesting chlorophyll-protein complex with a high fluorescence emission at 736 nm. Photobiochem Photobiophys. 1983;5:255-62.

86. Porra RJ, Thompson WA, Kriedemann PE. Determination of accurate extinction coefficients and simultaneous equations for assaying chlorophylls $a$ and $b$ extracted with four different solvents: verification of the concentration of chlorophyll standards by atomic absorption spectroscopy. Biochim Biophys Acta. 1989;975:384-94.

87. Quiles MJ, Garcia A, Cuello J. Separation by blue-native PAGE and identification of the whole $\mathrm{NAD}(\mathrm{P}) \mathrm{H}$ dehydrogenase complex from barley stroma thylakoids. Plant Physiol Biochem. 2000;38:225-32.

88. Candiano G, Bruschi M, Musante L, Santucci L, Ghiggeri GM, Carnemolla B, et al. Blue silver: a very sensitive colloidal Coomassie G-250 staining for proteome analysis. Electrophoresis. 2004;25:1327-33.

89. Solti Á, Müller B, Czech V, Sárvári É, Fodor F. Functional characterization of the chloroplast ferric chelate oxidoreductase enzyme. New Phytol. 2014;202:920-8

\section{Publisher's Note}

Springer Nature remains neutral with regard to jurisdictional claims in published maps and institutional affiliations.

Ready to submit your research? Choose BMC and benefit from:

- fast, convenient online submission

- thorough peer review by experienced researchers in your field

- rapid publication on acceptance

- support for research data, including large and complex data types

- gold Open Access which fosters wider collaboration and increased citations

- maximum visibility for your research: over $100 \mathrm{M}$ website views per year

At BMC, research is always in progress.

Learn more biomedcentral.com/submissions 